\title{
Leopold Herrmann
}

Periodic solutions of abstract differential equations: the Fourier method

Czechoslovak Mathematical Journal, Vol. 30 (1980), No. 2, 177-206

Persistent URL: http://dml.cz/dmlcz/101671

\section{Terms of use:}

(C) Institute of Mathematics AS CR, 1980

Institute of Mathematics of the Czech Academy of Sciences provides access to digitized documents strictly for personal use. Each copy of any part of this document must contain these Terms of use.

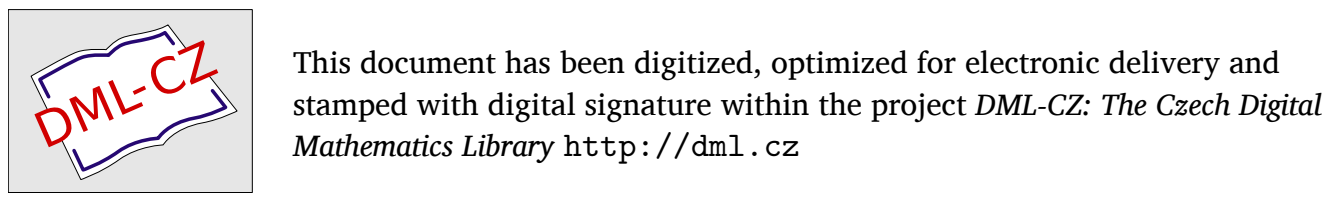




\title{
PERIODIC SOLUTIONS OF ABSTRACT DIFFERENTIAL EQUATIONS: THE FOURIER METHOD
}

\author{
Leopold Herrmann, Praha
}

(Received June 7, 1977)

\section{INTRODUCTION}

The development of the study of time-periodic solutions to partial differential equations of evolution arises in the thirties of this century. The generalizations to abstract differential equations appear subsequently. There exist today about 500 works concerning this subject. We refer the reader to the monograph VEJVODA ET AL. [15] where an extensive list of bibliography as well as a great number of various methods and approaches can be found.

The purpose of the present paper is to develop the Fourier method for finding periodic solutions of abstract differential equations of the first and second orders in $t$

$$
\begin{gathered}
u^{\prime}+(A+c) u=g+\varepsilon F(u) \\
u^{\prime \prime}+(a+b A) u^{\prime}+(A+c) u=g+\varepsilon F(u),
\end{gathered}
$$

where $A$ is a linear and $F$ a nonlinear operator, $a, b$ and $c$ are real constants and $\varepsilon \in\left[-\varepsilon_{0}, \varepsilon_{0}\right]$. For the theory and bibliography of $n$-th order abstract equations see STRAŠKRABA [13].

We shall look for a solution in the form of the Fourier series with respect to the system

$$
\left\{\mathrm{e}^{2 \pi \mathrm{i} j t / \omega} v_{k} ; j \in \mathbb{Z}, k \in \mathbb{N}\right\},
$$

where $v_{k}$ is the complete set of eigenfunctions of the operator $A$ and $\omega$ is a given period. In terms introduced in [15] we deal with the so called "time-space" Fourier method which belongs to the class of direct methods operating with periodic functions from the very beginning in contrast to the indirect ones where one solves first a related problem (for example, the corresponding initial-value or initial-boundary-value problem) and only then the $\omega$-periodicity is ensured. The well-known method (the "space"-Fourier method) when a solution is expanded with respect to $\left\{v_{k} ; k \in \mathbb{N}\right\}$ 
may serve as an example of the latter class. When expanding with respect to $\left\{\mathrm{e}^{2 \pi \mathrm{i} j t / \omega}\right.$; $j \in \mathbb{Z}\}$ we obtain the so called "time". Fourier method (which will be used in Section 8), another representant of direct methods.

It is convenient to work in Hilbert spaces since they offer both sufficiently simple and effective criteria for the convergence of series. We shall suppose that the operator $A$ (which is independent of $t$ ) is self-adjoint, bounded from below with a compact resolvent, or, without loss of generality, positive definite with $A^{-1}$ compact. The conditions imposed upon $A$ make it possible to develop a relatively simple theory which still covers a large number of situations encountered in mathematical physics. Let us note that a great advantage of this method consists in the fact that the original differential problem is reduced to an algebraic problem.

The preliminary Section 1 is concerned with periodic vector-valued functions. We define for them the Sobolev spaces and give their characterization by means of the behaviour of Fourier coefficients of the function with respect to the system $\left\{e^{2 \pi i j t / \omega} ; j \in \mathbb{Z}\right\}$. Let us remark that we deal with spaces of both integer and fractional orders, i.e., with both integer and fractional time derivatives.

In Section 2 we introduce the assumptions which play a role of basic importance in what follows. We state a lemma that forms a theoretical background of the Fourier method.

In the following three sections the equations $(0.1)$ and $(0.2)$ with $\varepsilon=0$ are investigated. For (0.2) the cases $|a|+|b|>0$ and $a=b=0$ are treated separately. We begin with the study of weak solutions, the results on the strong solvability are postponed to Section 7. In Sections 3-5 we use the (so called energetic) extension of the operator $A$ and the solutions are looked for in spaces of functions with finite energy (having a good physical meaning).

In Section 6 we indicate some simple methods how to use the results obtained for the linear case for the study of the (weakly) nonlinear problems (0.1) and (0.2).

The regularity of solutions may be investigated also by means of the time-space Fourier method. A basic tool in the proof is Lemma 7.2. Its application is easy but it should be noted that the results in concrete cases are not very favourable. Namely, we must work in domains of certain powers of the operator $A$. For example, if $A$ is a partial differential operator, $g, A g, A^{2} g, \ldots$ should fulfil (for a.e. $t$ ) the boundary conditions of the given problem (cf. [5]). The requirement that these conditions be satisfied is forced by the method used but it is needless for the existence of a smooth solution. We wish to have at our disposal a regularization theory which makes the use of these conditions unnecessary. Its development is presented in Section 8. Let us remark that some similar regularity results concerning the telegraph and wave equations are derived in [12].

Finally, in Section 9 the results are applied to partial differential equations with $A$ a second order elliptic operator. The theory can be applied also to the operators the eigenfunctions of which are orthogonal in a weight space. As an example, the Laplace operator in polar coordinates is considered. 
Throughout this paper we use the following symbols:

$\begin{array}{ll}\mathbb{C} & \text { the set of complex numbers } \\ \mathbb{R} & \text { the set of real numbers } \\ \mathbb{Z} & \text { the set of integers } \\ \mathbb{N} & \text { the set of positive integers } \\ \mathrm{cl} & \text { closure of a set } \\ \text { lin } & \text { linear hull } \\ \mathrm{G} & \text { embedding (algebraic and topologic) } \\ \mathscr{L}(X ; Y) & \text { space of linear continuous operators } L: X \rightarrow Y \\ \mathrm{dom} & \text { domain of definition of an operator } \\ \text { im } & \text { image (range) } \\ \mathrm{ker} & \text { kernel (null space) } \\ L \mid X X_{0} & \text { restriction of an operator } \\ \sim & \text { equivalence of norms } \\ X^{\prime} & \text { dual (antidual) to } X \\ {[X, Y]_{\Theta}} & \text { interpolation space (see [8]) } \\ P & \text { projection } \\ P^{c} & =I-P(I \text { identity operator) } \\ \omega & \text { a positive real number }- \text { period } \\ v & =2 \pi / \omega \\ U^{(r, s)}, U^{r, s} & \text { see p. } 195 \\ U_{M}^{(r, s)}, U_{M}^{r, s} & \text { see p. } 199 \\ \hat{U}^{(r, s)}, \hat{U}^{r, s} & \text { see p. } 183\end{array}$

\section{SOME FUNCTION SPACES}

Let $X$ be a Banach space.

By the symbol $\mathcal{C}(\omega ; X)$ we denote the space of functions $u: \mathbb{R} \rightarrow X$ that are $\omega$ periodic and continuous. Under the usual norm $C(\omega ; X)$ is a Banach space. Let $C^{\infty}(\omega ; X)$ be the space of $u: \mathbb{R} \rightarrow X \omega$-periodic infinitely differentiable. Defining the family of pseudonorms $p_{k}(u)=\max _{t \in[0, \omega]}\left\|u^{(k)}(t)\right\|_{X}, C^{\infty}(\omega ; X)$ is a local convex space.

Further, $\mathscr{D}^{\prime}(\omega ; X)=\mathscr{L}\left(C^{\infty}(\omega ; X) ; X_{w}\right)$ where $X_{w}$ stands for $X$ endowed with the weak topology. The elements of $\mathscr{D}^{\prime}(\omega ; X)$ are called periodic distributions. $\mathscr{D}^{\prime}(\omega ; X)$ is a local convex space (complete if $X$ is reflexive) if the family of pseudonorms is given by $p_{\varphi, f}(u)=|\langle f, u(\varphi)\rangle|, \varphi \in C^{\infty}(\omega ; \mathbb{R}), f \in X^{\prime}, u \in \mathscr{D}^{\prime}(\omega ; X)(\langle f, v\rangle$ means the dual pairing between the functional $f$ lying in $X^{\prime}$ and the element $v$ of $X$ ).

By $L_{1}(\omega ; X)$ we denote the space of (equivalence classes of) functions $u: \mathbb{R} \rightarrow X$ that are $\omega$-periodic and Bochner integrable over $(0, \omega) . L_{1}(\omega ; X)$ is a Banach space with the norm $\int_{0}^{\omega}\|u(t)\|_{X} \mathrm{~d} t$. 
Any function $u \in L_{1}(\omega ; X)$ determines a unique periodic distribution - denoted again by $u$ - by the rule $u(\varphi)=\int_{0}^{\omega} u(t) \varphi(t) \mathrm{d} t, \varphi \in C^{\infty}(\omega ; \mathbb{R})$; consequently, $L_{1}(\omega ; X) \subset \mathscr{D}^{\prime}(\omega ; X)$. For $u \in \mathscr{D}^{\prime}(\omega ; X)$ the distribution derivative is defined by $u^{\prime}(\varphi)=-u\left(\varphi^{\prime}\right), \varphi \in C^{\infty}(\omega ; \mathbb{P})$.

By $L_{2}(\omega ; X)$ (or sometimes $\left.W_{2}^{0}(\omega ; X)\right)$ we denote the space of (classes of) functions $u: \mathbb{R} \rightarrow X$ that are strongly measurable, $\omega$-periodic and

$$
\|u\|_{L_{2}(\omega ; X)}=\left(\int_{0}^{\omega}\|u(t)\|_{X}^{2} \mathrm{~d} t\right)^{1 / 2}<\infty .
$$

This norm makes $L_{2}(\omega ; X)$ a Banach space. We have $L_{2}(\omega ; X) \mathrm{G} L_{1}(\omega ; X)$.

For $r \in \mathbb{N}$, we denote by $W_{2}^{r}(\omega ; X)$ the space of (classes of) functions $u: \mathbb{P} \rightarrow X$ whose (distribution) derivatives up to the order $r$ belong to $L_{2}(\omega ; X) . W_{2}^{r}(\omega ; X)$ is a Banach space with the norm

$$
\|u\|_{W_{2}{ }^{r}(\omega ; X)}=\left(\sum_{k=0}^{r}\left\|u^{(k)}\right\|_{L_{2}(\omega ; X)}^{2}\right)^{1 / 2} .
$$

If $r$ is a positive non-integer then we define $W_{2}^{r}(\omega ; X)$ as a subspace of $W_{2}^{[r]}(\omega ; X)$ ( $[r]$ means the largest integer less than $r$ ) of (classes of) functions for which

$$
I(u)=\int_{0}^{\omega} \int_{0}^{\omega} \frac{\left\|u^{[r]}(t)-u^{[r]}(s)\right\|_{X}^{2}}{|t-s|^{1+2(r-[r])}} \mathrm{d} t \mathrm{~d} s<\infty .
$$

$W_{2}^{r}(\omega ; X)$ equipped with the norm

$$
\|u\|_{W_{2}^{r}(\omega ; X)}=\left(\|u\|_{W_{2}[r](\omega ; X)}^{2}+I(u)\right)^{1 / 2}
$$

is a Banach space.

Remark 1.1. We note that other equivalent definitions of the space $W_{2}^{1}(\omega ; X)$ (and of course of $W_{2}^{r}(\omega ; X), r \in \mathbb{N}$ ) are possible. For instance,

$$
\begin{gathered}
W_{2}^{1}(\omega ; X)=\left\{u ; u: \mathbb{P} \rightarrow X, \text { there is } v \in L_{2}(\omega ; X), \int_{0}^{\omega} v(t) \mathrm{d} t=0\right. \\
\text { and } \left.u(t)=u(0)+\int_{0}^{t} v(\tau) \mathrm{d} \tau\right\},
\end{gathered}
$$

or

$W_{2}^{1}(\omega ; X)=\{u ; u: \mathbb{R} \rightarrow X, u$ is $\omega$-periodic, locally absolutely continuous, the (strong) derivative $u^{\prime}(t)$ exists almost everywhere (the latter is a consequence of the absolute continuity in case of a reflexive $X)$ and $\left.u^{\prime} \in L_{2}(\omega ; X)\right\}$.

The spaces $W_{2}^{r}(\omega ; X)$ for $r$ non-integer can be defined by means of the interpolation. Our definition modifies that due to Aronszajn.

If $X$ is a Hilbert space, then $W_{2}^{r}(\omega ; X), r \geqq 0$, are also Hilbert spaces. 
Lemma 1.1. Let $X$ be a complex separable Hilbert space and $r \geqq 0$. Let $u \in$ $\in L_{2}(\omega ; X)$ and denote

$$
u_{j}=\omega^{-1 / 2} \int_{0}^{\omega} \mathrm{e}^{-\mathrm{i} v j t} u(t) \mathrm{d} t, \quad j \in \mathbb{Z}, \quad v=2 \pi / \omega .
$$

Then $u \in W_{2}^{r}(\omega ; X)$ if and only if

$$
\sum_{j=-\infty}^{\infty}\left(1+|j|^{2 r}\right)\left\|u_{j}\right\|_{X}^{2}<\infty .
$$

The condition being satisfied,

$$
u(t)=\omega^{-1 / 2} \sum_{j=-\infty}^{\infty} \mathrm{e}^{\mathrm{i} v j \mathrm{t}} u_{j},
$$

where the series converges in the sense of $W_{2}^{r}(\omega ; X)$. Moreover, the norm in $W_{2}^{r}(\omega ; X)$ is equivalent to that defined as the square root of the series in (1.1).

The proof for $r$ integer is easy and for $r$ non-integer presents a slight modification of the proof of Theorem 7.20 in [4].

Remark 1.2. The assumption of separability of $X$ is not substantial. The assertion suffices for our purposes since we shall deal only with separable Hilbert spaces (see Remark 2.1). Evidently, one can also relax the condition that $X$ is complex.

We conclude this section by some embedding theorems. Their proofs follow from Lemma 1.1 (Lemma 1.2, $1.3(\mathrm{a})$ ) or may be found in [7], [8].

Lemma 1.2. Let $X$ be a Hilbert space and $\delta>\frac{1}{2}$. Then

$$
W_{2}^{\delta}(\omega ; X) G C(\omega ; X)
$$

Lemma 1.3. Let $V$ and $H$ be two Hilbert spaces and $V G H G V^{\prime}$ densely. Then (a) $L_{2}(\omega ; V) \cap W_{2}^{2}\left(\omega ; V^{\prime}\right) G W_{2}^{1}(\omega ; H)$;

(b) $\left.L_{2}(\omega ; V) \cap W_{2}^{1}\left(\omega ; V^{\prime}\right) G C(\omega ; H)^{*}\right)$.

Lemma 1.4. Let $X_{0}, X$ and $X_{1}$ be Hilbert spaces, $X_{0} \mathrm{Q} X \mathrm{G} X_{1}$ and $X_{0} \mathrm{G} X$ compactly. Let $\delta>0$. Then $L_{2}\left(\omega ; X_{0}\right) \cap W_{2}^{\delta}\left(\omega ; X_{1}\right) G L_{2}(\omega ; X)$ compactly.

\section{BASIC ASSUMPTIONS}

Throughout the whole paper suppose that we are given

1. two (complex) Hilbert spaces $V$ and $H$ such that $V G H$ densely and compactly; 2. a continuous sesquilinear form $((\cdot, \cdot))$ on $V \times V$ that is $V$-elliptic and symmetric.

*) The norms on intersections of spaces are defined as usual. 
The form $((\cdot, \cdot))$ is an inner product on $V$. Without restricting generality we may assume the space $V$ normed by $\|u\|_{V}=((u, u))^{1 / 2}$.

Identifying $H$ with its antidual and denoting by $V^{\prime}$ the antidual to $V$, we obtain $V G H G V^{\prime}$ and both embeddings are dense. The inner product on $H$ as well as the dual pairing between $V^{\prime}$ and $V$ is denoted by the bracket $\langle\cdot, \cdot\rangle$.

The form $((\cdot, \cdot))$ determines a unique operator

$$
a: V \rightarrow V^{\prime}
$$

such that

$$
((u, v))=\langle a u, v\rangle, \quad u, v \in V .
$$

The following result is well-known.

Lemma 2.1 (Lax-Milgram). The operator $a$ is an isometric isomorphism of $V$ onto $V^{\prime}$.

Note that the inner product in $V^{\prime}$ satisfies $\langle u, v\rangle_{V^{\prime}}=\left(\left(a^{-1} u, a^{-1} v\right)\right), u, v \in V^{\prime}$. Denote $b=a^{-1} \mid V$. It is immediate that $b: V \rightarrow V$ is a positive symmetric compact operator. Using the well-known results on the spectrum of such operators we find that there exists a countable set of eigenvalues of $a$, the multiplicity of any eigenvalue is finite, the only limit point of the eigenvalues is $\infty$ and we can arrange them (taking into account their multiplicity) as follows:

$$
0<\lambda_{1} \leqq \lambda_{2} \leqq \lambda_{3} \leqq \ldots
$$

The corresponding system

$$
v_{1}, v_{2}, \ldots
$$

of eigenvectors can be chosen to form a complete orthonormal set in $H$. The systems

and

$$
\lambda_{1}^{-1 / 2} v_{1}, \lambda_{2}^{-1 / 2} v_{2}, \ldots
$$

$$
\lambda_{1}^{1 / 2} v_{1}, \lambda_{2}^{1 / 2} v_{2}, \ldots
$$

then form complete orthonormal sets in $V$ and $V^{\prime}$, respectively.

Remark 2.1. The above assumptions imply that the spaces $V$ and $H$ are separable.

The system

$$
\left\{\omega^{-1 / 2} \mathrm{e}^{\mathrm{i} v j t} v_{k} ; j \in \mathbb{Z}, k \in \mathbb{N}\right\}
$$

forms a complete orthonormal set in $L_{2}(\omega ; H)$ (and a complete set in $L_{2}(\omega ; V)$ and $\left.L_{2}\left(\omega ; V^{\prime}\right)\right)$.

The next lemma is of basic importance in the following sections. The spaces $W_{2}^{r}(\omega ; V), W_{2}^{r}(\omega ; H)(r \geqq 0)$ and $L_{2}\left(\omega ; V^{\prime}\right)$ will be characterized by means of the behaviour of Fourier coefficients of a function with respect to the system (2.1). For this purpose we introduce the following definition. 
For $r \geqq 0, s \geqq-1$ we denote by $\hat{O}^{(r, s)}$ the Hilbert space of sequences $\hat{u}=$ $=\left\{u_{j k} ; j \in \mathbb{Z}, k \in \mathbb{N}\right\}$ of complex. numbers such that

$$
\left.\|\hat{u}\|_{o(r, s)}=\left(\sum_{j, k}\left(1+|j|^{2 r}\right) \lambda_{k}^{s}\left|u_{j k}\right|^{2}\right)^{1 / 2}<\infty . *\right)
$$

Further, we set $\hat{U}^{r, s}=\hat{U}^{(r, 0)} \cap \hat{U}^{(0, s)}$.

Lemma 2.2. The mapping

$$
u \stackrel{\mathscr{g}}{\rightarrow}\left\{\omega^{-1 / 2} \int_{0}^{\omega}\left\langle u(t), v_{k}\right\rangle \mathrm{e}^{-\mathrm{i} v j t} \mathrm{~d} t ; j \in \mathbb{Z}, \quad k \in N\right\}
$$

is an isometric isomorphism of

(a) $L_{2}(\omega ; H)$ onto $\hat{U}^{0,0}$;

(b) $L_{2}(\omega ; V)$ onto $\hat{O}^{0,1}$;

(c) $L_{2}\left(\omega ; V^{\prime}\right)$ onto $\hat{U}^{0,-1}$

and a topological isomorphism of

(d) $W_{2}^{r}(\omega ; H)$ onto $\hat{U}^{r, 0}$,

(e) $W_{2}^{r}(\omega ; V)$ onto $\hat{U}^{(r, 1)}(r>0)$.

The inverse mapping is given by

$$
\left\{u_{j k} ; j \in \mathbb{Z}, k \in \mathbb{N}\right\} \stackrel{\mathscr{g}-1}{\longrightarrow} \omega^{-1 / 2} \sum_{j, k} u_{j k} \mathrm{e}^{\mathrm{i} v . j t} v_{k}
$$

(where the series converges in the sense of the respective space).

The proof of $(a)-(c)$ is immediate, for (d), (e) see Lemma 1.1.

\section{FIRST-ORDER EQUATIONS}

We shall deal with the following problem: let

$$
g \in L_{2}\left(\omega ; V^{\prime}\right) \text { and } c \in \mathbb{R}
$$

be given. We look for

$$
u \in L_{2}(\omega ; V)
$$

such that

$$
L_{1} u \equiv u^{\prime}+a u+c u=g .
$$

*) Here and hereafter $\sum_{j, k}$ means the summation over $j \in \mathbb{Z}$ and $k \in \mathbb{N}$. 
The equation (3.1) is meant in the sense of $\mathscr{D}^{\prime}\left(\omega ; V^{\prime}\right)$; hence it should hold

$$
\left\langle u^{\prime}(\varphi), w\right\rangle+\langle a u(\varphi), w\rangle+c\langle u(\varphi), w\rangle=\langle g(\varphi), w\rangle
$$

for all $\varphi \in C^{\infty}(\omega ; \mathbb{R})$ and $w \in V$.

Taking into account the equation (3.1), the assumption on $g$ and the fact that $a \in \mathscr{L}\left(V ; V^{\prime}\right)$ one obtains $u^{\prime} \in L_{2}\left(\omega ; V^{\prime}\right)$. (Thus we can establish the meaning of (3.1) in the point sense.)

The operator $L_{1}$ is defined on $L_{2}(\omega ; V) \cap W_{2}^{1}\left(\omega ; V^{\prime}\right)$ and obviously $L_{1} \in$ $\in \mathscr{L}\left(L_{2}(\omega ; V) \cap W_{2}^{1}\left(\omega ; V^{\prime}\right) ; L_{2}\left(\omega ; V^{\prime}\right)\right)$. In the sequel we shall formulate the results on solvability of the above problem via the properties of $L_{1}$ (and similarly in the following sections).

Remark 3.1. If $u$ is a solution then $u \in C(\omega ; H)$ by Lemma $1.3(\mathrm{~b})$.

Remark 3.2. The problem may be equivalently formulated by means of the integral identity. Indeed, a function $u$ is a solution if and only if $u \in L_{2}(\omega ; V)$ and

$$
-\int_{0}^{\omega} \overline{\left\langle v^{\prime}(t), u(t)\right\rangle} \mathrm{d} t+\int_{0}^{\omega}((u(t), v(t))) \mathrm{d} t+c \int_{0}^{\omega}\langle u(t), v(t)\rangle \mathrm{d} t=\int_{0}^{\omega}\langle g(t), v(t)\rangle \mathrm{d} t
$$

for all $v \in L_{2}(\omega ; V) \cap W_{2}^{1}\left(\omega ; V^{\prime}\right)$.

We shall look for a solution in the form of the Fourier series

$$
u(t)=\omega^{-1 / 2} \sum_{j, k} u_{j k} \mathrm{e}^{\mathrm{i} v j t} v_{k}
$$

where

$$
u_{j k}=\omega^{-1 / 2} \int_{0}^{\omega}\left\langle u(t), v_{k}\right\rangle \mathrm{e}^{\mathrm{i} v j t} \mathrm{~d} t .
$$

By Lemma 2.2, $u \in L_{2}(\omega ; V)$ if and only if

$$
\sum_{j, k} \lambda_{k}\left|u_{j k}\right|^{2}<\infty \text {. }
$$

The square root of this series defines the norm of $u$ in the space $L_{2}(\omega ; V)$.

Similarly,

$$
\begin{gathered}
g(t)=\omega^{-1 / 2} \sum_{j, k} g_{j k} \mathrm{e}^{\mathrm{i} v j t} v_{k}, \\
g_{j k}=\omega^{-1 / 2} \int_{0}^{\omega}\left\langle g(t), v_{k}\right\rangle \mathrm{e}^{-\mathrm{i} v j t} \mathrm{~d} t
\end{gathered}
$$

and

$$
\|g\|_{L_{2}\left(\omega ; V^{\prime}\right)}=\left(\sum_{j, k} \lambda_{k}^{-1}\left|g_{j k}\right|^{2}\right)^{1 / 2}<\infty .
$$

Inserting (3.2) and (3.5) into (3.1) we obtain easily the following system of equations for $u_{j k}$ :

$$
\left(\mathrm{i} v j+\lambda_{k}+c\right) u_{j k}=g_{j k}, \quad j \in \mathbb{Z}, \quad k \in \mathbb{N} .
$$


Let us distinguish two cases:

$$
c \neq-\lambda_{k} \text { for all } k \in \mathbb{N}
$$

$$
\begin{gathered}
\text { there is a } k_{0} \in \mathbb{N} \text { such that } \\
\lambda_{k_{0}-1}<-c=\lambda_{k_{0}}=\lambda_{k_{0}+1}=\ldots=\lambda_{k_{0}+m-1}<\lambda_{k_{0}+m} \quad\left(\lambda_{0} \equiv 0\right),
\end{gathered}
$$

i.e., $-c$ is an eigenvalue of $a$ of multiplicity $m$.

In the case (3.8)

$$
u_{j k}=\left(\mathrm{i} v j+\lambda_{k}+c\right)^{-1} g_{j k}, \quad j \in \mathbb{Z}, \quad k \in \mathbb{N}
$$

which yields

$$
\begin{aligned}
\|u\|_{L_{2}(\omega ; V)}^{2} & =\sum_{j, k} \lambda_{k}\left|u_{j k}\right|^{2}=\sum_{j, k} \lambda_{k}\left(v^{2} j^{2}+\left(\lambda_{k}+c\right)^{2}\right)^{-1}\left|g_{j k}\right|^{2} \leqq \\
\leqq & \text { const. } \sum_{j, k} \lambda_{k}^{-1}\left|g_{j k}\right|^{2}=\text { const. }\|g\|_{L_{2}\left(\omega ; V^{\prime}\right)}^{2} .
\end{aligned}
$$

The series (3.2) with $u_{j k}$ defined by (3.10) gives the only solution of our problem. Evidently, $\left\|u^{\prime}\right\|_{L_{2}\left(\omega ; V^{\prime}\right)} \leqq$ const. $\|g\|_{L_{2}\left(\omega ; V^{\prime}\right)}$. We have proved that the operator $L_{1}$ is an isomorphism of $L_{2}(\omega ; V) \cap W_{2}^{1}\left(\omega ; V^{\prime}\right)$ onto $L_{2}\left(\omega ; V^{\prime}\right)$.

If (3.9) takes place, then in view of the formula for $u_{j k}$

where

$$
\operatorname{ker} L_{1}=\operatorname{lin}\left\{\mathrm{e}^{\mathrm{i} v j t} v_{k} ;(j, k) \in \mathscr{S}_{1}\right\}
$$

and the necessary condition of solvability is $P g=0$ if $P$ denotes the orthogonal projection of $L_{2}\left(\omega ; V^{\prime}\right)$ onto the subspace $\operatorname{lin}\left\{\mathrm{e}^{\mathrm{i} v / t} v_{k} ;(j, k) \in \mathscr{S}_{1}\right\}$. It is even sufficient since the above estimates yield the existence of the continuous right inverse operator $L_{1}^{-1}=\left(L_{1} \mid \operatorname{dom} L_{1} \cap \operatorname{im} P^{c}\right)^{-1}: \operatorname{im} P^{c} \rightarrow \operatorname{dom} L_{1} \cap \operatorname{im} P^{c}$. (In what follows, the inverse as well as the right inverse operator to $L_{n}, n=1,2,3,4$, will be denoted by $L_{n}^{-1}$.) The general solution of the problem is given by $u=v+w$, where $v \in \operatorname{ker} L_{1}, w$ is the unique solution from the subspace orthogonal to $\operatorname{ker} L_{1}$ and is given by $w=L_{1}^{-1} g$.

Summarizing our results we get the following theorem.

Theorem 3.1. The operator $L_{1}$ is a Fredholm mapping of index zero*) for all $c \in \mathbb{R}$. More precisely:

1) Let (3.8) hold. Then

$$
L_{1}^{-1} \in \mathscr{L}\left(L_{2}\left(\omega ; V^{\prime}\right) ; L_{2}(\omega ; V) \cap W_{2}^{1}\left(\omega: V^{\prime}\right)\right)
$$

*) I.e., im $L_{1}$ is closed and $\operatorname{dim} \operatorname{ker} L_{1}=\operatorname{codim} \operatorname{im} L_{1}<\infty$. 
2) Let (3.9) hold. Then

and

$$
\operatorname{ker} L_{1}=L_{2}(\omega ; V) \cap \operatorname{im} P, \quad \operatorname{dim} \operatorname{ker} L_{1}=m
$$

$$
L_{1}^{-1} \in \mathscr{L}\left(L_{2}\left(\omega ; V^{\prime}\right) \cap \operatorname{im} P^{c} ; L_{2}(\omega ; V) \cap W_{2}^{1}\left(\omega ; V^{\prime}\right) \cap \operatorname{im} P^{c}\right),
$$

where $P$ is the orthogonal projection $L_{2}\left(\omega ; V^{\prime}\right)$ onto $\operatorname{lin}\left\{v_{k_{0}}, \ldots, v_{k_{0}+m-1}\right\}$.

Remark 3.3. The case of non-trivial kernel is said to be critical (or resonance), the opposite case is said to be non-critical (non-resonance).

\section{SECOND-ORDER EQUATIONS WITH DISSIPATIVE TERMS}

We are interested in solving the equation

$$
u^{\prime \prime}+(a+b a) u^{\prime}+(a+c) u=g
$$

where $a, b, c \in \mathbb{R}$ and $|a|+|b|>0$.

CASE $1: b \neq 0$.

It is to find

$$
u \in W_{2}^{1}(\omega ; V)
$$

such that

$$
L_{2} u \equiv u^{\prime \prime}+(a+b a) u^{\prime}+(a+c) u=g,
$$

where $g \in L_{2}\left(\omega ; V^{\prime}\right)$.

The equation is meant in the sense of $\mathscr{D}^{\prime}\left(\omega ; V^{\prime}\right)$. If $u$ is a solution, then $u^{\prime \prime} \in$ $\in L_{2}\left(\omega ; V^{\prime}\right)$. Evidently $L_{2} \in \mathscr{L}\left(W_{2}^{1}(\omega ; V) \cap W_{2}^{2}\left(\omega ; V^{\prime}\right) ; L_{2}\left(\omega ; V^{\prime}\right)\right)$.

Remark 4.1. A function $u$ is a solution if and only if $u \in W_{2}^{1}(\omega ; V)$ and

$$
\begin{gathered}
-\int_{0}^{\omega}\left\langle u^{\prime}(t), v^{\prime}(t)\right\rangle \mathrm{d} t+\int_{0}^{\omega}\left(\left(b u^{\prime}(t)+u(t), v(t)\right)\right) \mathrm{d} t+\int_{0}^{\omega}\left\langle a u^{\prime}(t)+c u(t), v(t)\right\rangle \mathrm{d} t= \\
=\int_{0}^{\omega}\langle g(t), v(t)\rangle \mathrm{d} t
\end{gathered}
$$

for all $v \in L_{2}(\omega ; V) \cap W_{2}^{1}(\omega ; H)$.

Remark 4.2. If $u$ is a solution, then $u \in C(\omega ; V)$ and $u^{\prime} \in C(\omega ; H)$, by Lemma 1.2 and $1.3(\mathrm{~b})$.

We look for a solution again in the form (3.2). By Lemma 2.2, $u \in W_{2}^{-1}(\omega ; V)$ if and only if

$$
\|u\|_{W_{2}{ }^{1}(\omega ; V)}^{2} \sim \sum_{j, k}\left(1+j^{2}\right) \lambda_{k}\left|u_{j k}\right|^{2}<\infty .
$$


Using the expansion (3.5) we arrive at the system for $u_{j k}$

$$
\left(-v^{2} j^{2}+\lambda_{k}+c+\mathrm{i} v j\left(a+b \lambda_{k}\right)\right) u_{j k}=g_{j k}, \quad j \in \mathbb{Z}, \quad k \in \mathbb{N} .
$$

The coefficient at $u_{j k}$ vanishes if and only if either (3.9) holds or $\lambda_{k}=-a / b$ and $(c b-a) / b v^{2}$ is a perfect square. Denote

$$
\mathscr{S}_{2}=\mathscr{S}_{1} \cup\left\{(j, k) ;(j, k) \in \mathbb{Z} \times \mathbb{N}, \lambda_{k}=-\frac{a}{b}, \quad j^{2}=\frac{c b-a}{b v^{2}}\right\}
$$

(for $\mathscr{S}_{1}$ see (3.11)). Taking into account the estimate

$$
\frac{\left(1+j^{2}\right) \lambda_{k}}{\left(-v^{2} j^{2}+\lambda_{k}+c\right)^{2}+v^{2} j^{2}\left(a+b \lambda_{k}\right)^{2}} \leqq \frac{\text { const. }}{\lambda_{k}}, \quad(j, k) \in(\mathbb{Z} \times \mathbb{N}) \backslash \mathscr{S}_{2},
$$

(4.2) and (3.7), we obtain the following result.

Theorem 4.1. The operator $L_{2}$ is a Fredholm mapping of index zero for all $a, b, c \in \mathbb{R}, b \neq 0$.

More precisely:

1) Let $\mathscr{S}_{2}=\emptyset$. Then

$$
L_{2}^{-1} \in \mathscr{L}\left(L_{2}\left(\omega ; V^{\prime}\right) ; W_{2}^{1}(\omega ; V) \cap W_{2}^{2}\left(\omega ; V^{\prime}\right)\right) .
$$

2) Let $\mathscr{S}_{2} \neq \emptyset$. Then

and

$$
\operatorname{ker} L_{2}=W_{2}^{1}(\omega ; V) \cap \operatorname{im} P, \quad \operatorname{dim} \operatorname{ker} L_{2}=\operatorname{card} \mathscr{S}_{2}<\infty
$$

$$
L_{2}^{-1} \in \mathscr{L}\left(L_{2}\left(\omega ; V^{\prime}\right) \cap \operatorname{im} P^{c} ; \quad W_{2}^{1}(\omega ; V) \cap W_{2}^{2}\left(\omega ; V^{\prime}\right) \cap \operatorname{im} P^{c}\right),
$$

where $P$ is the orthogonal projection of $L_{2}\left(\omega ; V^{\prime}\right)$ onto $\operatorname{lin}\left\{\mathrm{e}^{\mathrm{i} v j t} v_{k} ;(j, k) \in \mathscr{S}_{2}\right\}$.

CASE 2: $b=0$ (hence $a \neq 0$ ).

We treat the problem of finding

such that

$$
u \in L_{2}(\omega ; V) \cap W_{2}^{1}(\omega ; H)
$$

$$
L_{3} u \equiv u^{\prime \prime}+a u^{\prime}+a u+c u=g,
$$

where $g \in L_{2}\left(\omega ; V^{\prime}\right)$.

A function $u$ being a solution satisfies $u^{\prime \prime} \in L_{2}\left(\omega ; V^{\prime}\right)$. The operator $L_{3}$ belongs to the space

$$
\mathscr{L}\left(L_{2}(\omega ; V) \cap W_{2}^{2}\left(\omega ; V^{\prime}\right) ; L_{2}\left(\omega ; V^{\prime}\right)\right)
$$


(cf. Lemma 1.3 (a)). As we shall see later (cf. Example 4.1) the present problem is in general not solvable (not even in the non-critical case) for $g \in L_{2}\left(\omega ; V^{\prime}\right)$, but only for $g \in L_{2}(\omega ; H)$. For simplicity we introduce the following notation:

$$
\begin{aligned}
& U^{1,1}=L_{2}(\omega ; V) \cap W_{2}^{1}(\omega ; H) \\
& U^{0,0}=L_{2}(\omega ; H) .
\end{aligned}
$$

Remark 4.3. If $u \in U^{1,1}$ solves (4.4) then $u \in C(\omega ; H)$ and $u^{\prime} \in C\left(\omega ; V^{\prime}\right)$.

Writing $u$ and $g$ in the form (3.2) and (3.5), respectively, and using the same technique as before one obtains

$$
\left(-v^{2} j^{2}+\lambda_{k}+c+\mathrm{i} v j a\right) u_{j k}=g_{j k}, \quad j \in \mathbb{Z}, \quad k \in \mathbb{N} .
$$

Lemma 4.1. To any $\varepsilon \in(0,1)$ and $r \in[1,2]$ there exists $C>0$ such that

$$
\frac{j^{2 r}+\lambda_{k}^{r}}{\left(-v^{2} j^{2}+\lambda_{k}+c\right)^{2}} \leqq \frac{C}{\lambda_{k}^{2-r}}, \quad(j, k) \in(\mathbb{Z} \times \mathbb{N}) \backslash \Xi^{e},
$$

where

$$
\begin{gathered}
\Xi^{\varepsilon}=\{(j, k) ;(j, k) \in \mathbb{Z} \times \mathbb{N}, \text { there exists } \Theta \in(1-\varepsilon, 1+\varepsilon), \\
\left.v|j|=\Theta\left(\lambda_{k}+c\right)^{1 / 2}\right\} .
\end{gathered}
$$

Proof. There is $k^{+} \in \mathbb{N}$ such that $\lambda_{k}+c \leqq 0$ for $k=1, \ldots, k^{+}-1$ and $\lambda_{k}+c>$ $>0$ for $k \geqq k^{+}$. Further, there are two constants $c_{1}>0$ and $c_{2}>0$ such that

$$
c_{1} \lambda_{k} \leqq \lambda_{k}+c \leqq c_{2} \lambda_{k}, \quad k \geqq k^{+} .
$$

Denote $\Xi_{0}=\mathbb{Z} \times\left\{1, \ldots, k^{+}-1\right\}$. For $\Theta \geqq 0$ denote $R_{\Theta}=\{(j, k) ;(j, k) \in \mathbb{Z} \times \mathbb{N}$, $\left.k \geqq k^{+}, v|j|=\Theta\left(\lambda_{k}+c\right)^{1 / 2}\right\}$. We have $\mathbb{Z} \times\left(\mathbb{N} \backslash\left\{1, \ldots, k^{+}-1\right\}\right)=\bigcup_{\theta \in[0, \infty)} R_{\theta}$. Let $\varepsilon \in(0,1)$. Put $\Xi_{1}^{\varepsilon}=\bigcup_{\theta \in[0,1-\varepsilon]} R_{\Theta}, \Xi^{\varepsilon}=\bigcup_{\Theta \in(1-\varepsilon, 1+\varepsilon)} R_{\Theta}, \Xi_{2}^{\varepsilon}=\bigcup_{\Theta \in[1+\varepsilon, \infty)} R_{\Theta}$. We get

$$
\begin{aligned}
& a_{j k}^{(r)} \equiv \frac{j^{2 r}+\lambda_{k}^{r}}{\left(-v|j|+\left(\lambda_{k}+c\right)^{1 / 2}\right)^{2}\left(v|j|+\left(\lambda_{k}+c\right)^{1 / 2}\right)^{2}} \leqq \\
& \leqq \text { const. } \frac{\Theta^{2 r}\left(\lambda_{k}+c\right)^{r}+\lambda_{k}^{r}}{\varepsilon^{2}(\Theta+1)^{2}\left(\lambda_{k}+c\right)^{2}} \leqq \frac{\text { const. }}{\lambda_{k}^{2-r}}, \quad(j, k) \in \Xi_{1}^{\varepsilon}
\end{aligned}
$$

and

$$
\begin{gathered}
a_{j k}^{(r)} \leqq \text { const. } \frac{\Theta^{2 r}\left(\lambda_{k}+c\right)^{r}+\lambda_{k}^{r}}{\varepsilon^{2}(1+\varepsilon)^{-2} \Theta^{2}(\Theta+1)^{2}\left(\lambda_{k}+c\right)^{2}} \leqq \\
\leqq \text { const. } \frac{1+\Theta^{-4}}{\left(1+\Theta^{-1}\right)^{2} \lambda_{k}^{2-r}} \leqq \frac{\text { const. }}{\lambda_{k}^{2-r}}, \quad(j, k) \in \Xi_{2}^{\varepsilon}
\end{gathered}
$$

and the proof follows immediately. 
Using this lemma with $r=1$, taking into account that " $j^{2}$ behave as $\lambda_{k}$ on $\Xi^{\varepsilon "}$ we find

$$
\frac{j^{2}+\lambda_{k}}{\left(-v^{2} j^{2}+\lambda_{k}+c\right)^{2}+a^{2} v^{2} j^{2}} \leqq \text { const. }
$$

for $(j, k) \in \mathbb{Z} \times \mathbb{N}$ or merely for $(j, k) \in(\mathbb{Z} \times \mathbb{N}) \backslash \mathscr{S}_{1}$ if $(3.9)$ holds. Since

and

$$
\|u\|_{U^{1,1}}^{2} \sim \sum_{j, k}\left(j^{2}+\lambda_{k}\right)\left|u_{j k}\right|^{2}
$$

we can state the following theorem.

$$
\|g\|_{U^{0,0}}^{2}=\sum_{j, k}\left|g_{j k}\right|^{2}
$$

Theorem 4.2. 1) Let (3.8) hold. Then

$$
L_{3}^{-1} \in \mathscr{L}\left(U^{0,0} ; U^{1,1} \cap W_{2}^{2}\left(\omega ; V^{\prime}\right)\right) \text {. }
$$

2) Let (3.9) hold. Then

and

$$
\operatorname{ker} L_{3}=U^{1,1} \cap \operatorname{im} P, \operatorname{dim} \operatorname{ker} L_{3}=m
$$

$$
L_{3}^{-1} \in \mathscr{L}\left(U^{0,0} \cap \operatorname{im} P^{c} ; U^{1,1} \cap W_{2}^{2}\left(\omega ; V^{\prime}\right) \cap \operatorname{im} P^{c}\right)
$$

where $P$ is the orthogonal projection of $U^{0,0}$ onto lin $\left\{v_{k_{0}}, \ldots, v_{k_{0}+m-1}\right\}$.

Example 4.1. The operator $L_{2}$ (similarly as $L_{1}$ ) has always a closed range in $L_{2}\left(\omega ; V^{\prime}\right)$. For $L_{3}$ this is no longer true, although (say, in the non-critical case) cl im $L_{3}=L_{2}\left(\omega ; V^{\prime}\right)$. To see an example, let $H=L_{2}(0, \pi)$,

$$
V=\stackrel{\circ}{W}_{2}^{1}(0, \pi), \quad((u, v))=\int_{0}^{\pi} u^{\prime}(x) \overline{v^{\prime}(x)} \mathrm{d} x, \text { hence } \lambda_{k}=k^{2}
$$

and $v_{k}=(2 / \pi)^{1 / 2} \sin k x$. Define $g$ by the formula (3.5) where

$$
g_{j k}=0, \quad|j| \neq k, \quad g_{-k, k}=g_{k k}=k^{-1 / 2}, \quad k \in \mathbb{N} .
$$

It is easy to check that $g \in L_{2}\left(2 \pi ; W_{2}^{-1}(0, \pi)\right)$ and that our problem with $\omega=2 \pi$, $c=0$ has no solution in the space $U^{1,1}$.

\section{SECOND-ORDER EQUATIONS WITHOUT DISSIPATIVE TERMS}

We shall investigate the problem:

$$
\begin{gathered}
u \in U^{1,1}, \\
L_{4} u \equiv u^{\prime \prime}+a u+c u=g,
\end{gathered}
$$

where $g \in U^{0,0}$ and $c \in \mathbb{R}$. (For $U^{1,1}$ and $U^{0,0}$ see (4.6) and (4.7).) 
The operator $L_{4}$ belongs to (4.5) and we could give again an equivalent formulation of the problem in terms of the integral identity.

The solution is sought again in the form (3.2). In contrast to the equation (4.4) the condition $g \in U^{0,0}$ is not sufficient, in general, to guarantee the solvability of the present problem. We shall assume that $g$ lies in the space

$$
U^{r, 0}=W_{2}^{r}(\omega ; H)
$$

where $r \geqq 0$ will be prescribed later. Writing $g$ in the form (3.5) we have by Lemma 2.2

$$
\|g\|_{U^{r, o}} \sim \sum_{j, k}\left(1+|j|^{2 r}\right)\left|g_{j k}\right|^{2}<\infty .
$$

The Fourier coefficients $u_{j k}$ are defined by

$$
\left(-v^{2} j^{2}+\lambda_{k}+c\right) u_{j k}=g_{j k}, \quad j \in \mathbb{Z}, \quad k \in \mathbb{N} .
$$

Denote

$$
\mathscr{S}_{4}=\left\{(j, k) ;(j, k) \in \mathbb{Z} \times \mathbb{N},-v^{2} j^{2}+\lambda_{k}+c=0\right\} .
$$

Putting

$$
a_{j k}=\left(-v^{2} j^{2}+\lambda_{k}+c\right)^{-2}\left(j^{2}+\lambda_{k}\right)
$$

and using the notation from the proof of Lemma 4.1 we get easily that the set $\left\{\lambda_{k} a_{j k} ;(j, k) \in \Xi_{0} \cup \Xi_{1}^{\varepsilon} \cup \Xi_{2}^{\varepsilon}\right\}$ is bounded. Further investigation on $\Xi^{\varepsilon}$ depends essentialy on the behaviour of the eigenvalues $\lambda_{k}$ near infinity and on their relation to the numbers $v^{2} j^{2}-c$ (this is due to the absence of the dissipative term $a u^{\prime}$ in comparison with the problem (4.4)). We make the assumption

$\left(\Lambda_{\beta}\right)$ there are constants $M>0$ and $\beta \geqq 0$ such that

$$
\begin{gathered}
\left|\left(\lambda_{k}+c\right)^{1 / 2}-v\right| j|| \geqq M|j|^{-\beta} \quad\left(\text { resp. } M \lambda_{k}^{-\beta / 2}\right) \\
(j, k) \in(\mathbb{Z} \times \mathbb{N}) \backslash \mathscr{S}_{4}, \quad \lambda_{k}+c>0 .
\end{gathered}
$$

Making use of $\left(\Lambda_{\beta}\right)$ and (4.8) we get

$$
a_{j k} \leqq \text { const. } \frac{\Theta^{2}\left(\lambda_{k}+c\right)+\lambda_{k}}{(\Theta+1)^{2}\left(\lambda_{k}+c\right) M^{2}}|j|^{2 \beta} \leqq \text { const. }|j|^{2 \beta}, \quad(j, k) \in \Xi^{e} \backslash \mathscr{S}_{4} .
$$

Consequently,

$$
a_{j k} \leqq \text { const. }\left(1+|j|^{2 \beta}\right), \quad(j, k) \in(\mathbb{Z} \times \mathbb{N}) \backslash \mathscr{S}_{4} .
$$

Hence $u \in U^{1,1}$ if $g \in U^{\beta, 0}$ and $g_{j k}=0$ for $(j, k) \in \mathscr{S}_{4}$.

Let us summarize the results in the usual formulation. 
Theorem 5.1. Let the condition $\left(\Lambda_{\beta}\right)$ be satisfied.

1) Let $\mathscr{S}_{4}=\emptyset$. Then

$$
L_{4}^{-1} \in \mathscr{L}\left(U^{\beta, 0} ; U^{1,1} \cap W_{2}^{2}\left(\omega ; V^{\prime}\right)\right)
$$

2) Let $\mathscr{S}_{4} \neq \emptyset$. Then

and

$$
\begin{gathered}
\operatorname{dim} \operatorname{ker} L_{4}=\operatorname{card} \mathscr{S}_{4}, \\
\operatorname{ker} L_{4}=U^{1,1} \cap W_{2}^{2}\left(\omega ; V^{\prime}\right) \cap \operatorname{im} P
\end{gathered}
$$

$$
L_{4}^{-1} \in \mathscr{L}\left(U^{\beta, 0} \cap \operatorname{im} P^{c} ; U^{1,1} \cap W_{2}^{2}\left(\omega ; V^{\prime}\right) \cap \operatorname{im} P^{c}\right),
$$

where $P$ is the orthogonal projection of $U^{0,0}$ onto $\mathrm{cl} \operatorname{lin}\left\{\mathrm{e}^{\mathrm{i} v j t} v_{k} ;(j, k) \in \mathscr{S}_{4}\right\}$.

Remark 5.1. Let us remark explicitly that here, in contrast to the problems considered in the previous paragraphs, the kernel may be infinite-dimensional.

Remark 5.2. A similar approach may be used for the first-order equations of the "Schrödinger type"

$$
i u^{\prime}+a u+c u=g .
$$

In the end, we illustrate the theory by a few examples. They will suggest that even in very simple situations one has to treat rather difficult number-theoretical problems. We suppose $c=0$.

Example 5.1. Let $H=L_{2}(0, \pi), V=\stackrel{\circ}{W}_{2}^{1}(0, \pi),((u, v))=\int_{0}^{\pi} u^{\prime}(x) \overline{v^{\prime}(x)} \mathrm{d} x$. Here $\lambda_{k}=k^{2}, k \in \mathbb{N}$. Let $\omega=2 \pi p / q$, where $p, q \in \mathbb{N}$ are relatively prime. We check easily that $\mathscr{S}_{4}=\{(j, k) ;(j, k) \in \mathbb{Z} \times \mathbb{N},-|j| q \mid p+k=0\}=\{(j, k) ;|j|=n p$, $k=n q, n \in \mathbb{N}\}$. The condition $\Lambda_{\beta}$ is fulfilled with $\beta=0$, for

$$
|k-| j|q| p \mid \geqq 1 / p, \quad(j, k) \in(\mathbb{Z} \times \mathbb{N}) \backslash \mathscr{S}_{4} .
$$

Example 5.2. Let $H=L_{2}(0, \pi), V=W_{2}^{2}(0, \pi) \cap \dot{W}_{2}^{1}(0, \pi),((u, v))=\int_{0}^{\pi} u^{\prime \prime}(x)$. $\overline{v^{\prime \prime}(x)} \mathrm{d} x$. Now $\lambda_{k}=k^{4}, k \in \mathbb{N}$. Let again $\omega=2 \pi p / q, p, q \in \mathbb{N}$ relatively prime.

We prove (following [15]) that $\mathscr{S}_{4}=\left\{(j, k) ;(j, k) \in \mathbb{Z} \times \mathbb{N},-|j| q \mid p+k^{2}=\right.$ $=0\}=\left\{(j, k) ;|j|=n^{2} p q_{1}, k=n q_{1} q_{2}, n \in \mathbb{N}\right\}$ while we have expressed $q$ in the form $q=q_{1} q_{2}^{2}$, where $q_{1}$ is not divisible by a square of an integer greater than 1 . For $(j, k) \in \mathscr{S}_{4}$ we have $k^{2} p=|j| q$ which means that $|j| q$ is divisible by $p$. Since $p, q$ are relatively prime, there is $j^{\prime} \in \mathbb{N}$ such that $|j|=j^{\prime} p$, hence $k^{2}=j^{\prime} q$. Writing $q$ in the above form $q_{1} q_{2}^{2}$ we see that $q_{2}$ divides $k$ and setting $k=k^{\prime} q_{2}$ for some $k^{\prime} \in \mathbb{N}$, we easily find that $q_{1}$ divides $k^{\prime}, k^{\prime}=n q_{1}$ for some $n \in \mathbb{N}$. Hence $k=n q_{1} q_{2}$ and therefore $|j|=n^{2} p q_{1}$ for some $n \in \mathbb{N}$. On the other hand, if $k$ and $j$ satisfy the above relations, then $(j, k) \in \mathscr{S}_{4}$.

The condition $\left(\Lambda_{\beta}\right)$ is evidently fulfilled with $\beta=0$.

Example 5.3. Let the spaces and the form be the same as in Example 5.1. Let $\omega=2 \pi \alpha$ where $\alpha$ is irrational. Then $\mathscr{S}_{4}=\emptyset$. The condition $\left(\Lambda_{\beta}\right)$ is fulfilled if there 
is a constant $C>0$ such that

$$
|\alpha-l| k \mid \geqq C k^{-1-\beta / 2}, \quad l, k \in \mathbb{N} .
$$

This is true, for instance, in the case that $\alpha$ is an algebraic number of degree $1+$ $+\beta / 2(\in \mathbb{N})$ (see [3], p. 42).

It may be shown that there exists an uncountable dense in $\mathbb{R}$ set of measure zero of irrational numbers $\alpha$ which satisfy (5.4) with $\beta=2$ (and $C$ depending on $\alpha$ ). The inequality

$$
|\alpha-l / k| \geqq C(\alpha) k^{-2} \log ^{-1-\varepsilon}(k+1)
$$

is fulfilled by almost all irrational numbers $\alpha$.

The results of Novík [10] yield the following assertion: we let $\gamma=\gamma(\alpha)$ be the supremum of all $\beta$ for which the inequality $|-l / \alpha+k|<l^{-\beta}$ has infinitely many solutions for $l, k \in \mathbb{N}$. Let $\delta \geqq 0$. Then

a) if $\gamma<\delta$ then for any $g \in W_{2}^{\delta}(\omega ; H)$ there is a solution of the problem in $U^{1,1}$,

b) if $\gamma>\delta$ then there exists a function $g \in W_{2}^{\delta}(\omega ; H)$ such that the problem has no solution in $U^{1,1}$.

\section{THE NONLINEAR CASE}

The results obtained in the preceding sections may be used for the study of nonlinear problems

$$
L_{n} u=F(u)
$$

or weakly nonlinear problems

$$
L_{n} u=\varepsilon F(u, \varepsilon), \quad \varepsilon \in\left[-\varepsilon_{0}, \varepsilon_{0}\right]\left(\varepsilon_{0}>0\right)
$$

$(n=1,2,3,4)$.

Our aim is to present here only three simple methods. They are based on the Schauder or Banach fixed point theorems (in the non-critical cases) and on the implicit function theorem (in the critical cases). It should be noted that the problems of this kind attract a great deal of interest today and have recently been studied by a number of authors (see [15] for references).

The results will be formulated explicitly only for $L_{1}$ (with the exception - Theorem 6.4 which involves $L_{3}$ ). We leave analogous formulations of results for the other operators to the reader.

For simplicity of notation we set $\mathscr{U}=L_{2}(\omega ; V) \cap W_{2}^{1}\left(\omega ; V^{\prime}\right)$.

Theorem 6.1. Suppose (3.8) holds. Let the following assumptions be satisfied:

a) $V_{1}$ is a Hilbert space, $V \mathrm{G} V_{1} G V^{\prime}$ and $V \mathrm{G} V_{1}$ compactly;

b) $F$ maps continuously $L_{2}\left(\omega ; V_{1}\right)$ into $L_{2}\left(\omega ; V^{\prime}\right)$;

c) there are three constants $c_{1}>0, c_{2}>0$ and $\sigma \in[0,1]$ such that

$$
\|F(u)\|_{L_{2}\left(\omega ; V^{\prime}\right)} \leqq c_{1}+c_{2}\|u\|_{\mathscr{U}}^{\sigma}, \quad u \in \mathscr{U}
$$


(and if $\sigma=1$ then, moreover, $c_{2}$ is sufficiently small). Then there exists $u \in \mathscr{U}$ such that

$$
L_{1} u=F(u) \text {. }
$$

The proof is an easy consequence of the Schauder fixed point theorem and of Lemma 1.4.

On the other hand, our next result follows easily from the Banach contraction principle.

Theorem 6.2. Suppose (3.8) holds. Let the following assumptions be satisfied:

a) there are $\varrho>0$ and $\varepsilon_{0}>0$ such that for $u \in \mathscr{U}$ with $\|u\|_{\mathscr{U}} \leqq \varrho, \varepsilon \rightarrow F(u, \varepsilon)$ is a continuous mapping of $\left[-\varepsilon_{0}, \varepsilon_{0}\right]$ into $L_{2}\left(\omega ; V^{\prime}\right)$,

b) there is $\lambda>0$ such that $\left\|F\left(u_{1}, \varepsilon\right)-F\left(u_{2}, \varepsilon\right)\right\|_{L_{2}\left(\omega ; V^{\prime}\right)} \leqq \lambda\left\|u_{1}-u_{2}\right\|_{\mathscr{\ell}},\left\|u_{1}\right\|_{\mathscr{U}_{\ell}} \leqq \varrho$, $\left\|u_{2}\right\|_{\mathcal{u}} \leqq \varrho, \varepsilon \in\left[-\varepsilon_{0}, \varepsilon_{0}\right]$.

Then there is $\varepsilon^{*} \in\left(0, \varepsilon_{0}\right]$ such that for any $\varepsilon \in\left[-\varepsilon^{*}, \varepsilon^{*}\right]$ there is a unique $u(\varepsilon) \in \mathscr{U}$ satisfying

$$
L_{1} u(\varepsilon)=\varepsilon F(u(\varepsilon), \varepsilon)
$$

Moreover, $\varepsilon \rightarrow u(\varepsilon)$ is a continuous mapping of $\left[-\varepsilon^{*}, \varepsilon^{*}\right]$ into $\mathcal{U}$.

It is easy to see that if we can solve the equation (6.1) we are able also to solve the equation

$$
L_{1} u(\varepsilon)=g+F(u(\varepsilon), \varepsilon)
$$

We now turn to the critical case. Here the alternative method is used frequently. The solution of (6.1) is looked for in the form

$$
u(\varepsilon)=v(\varepsilon)+w(\varepsilon)
$$

where $v(\varepsilon) \in \mathscr{U} \cap \operatorname{im} P, w(\varepsilon) \in \mathscr{U} \cap \operatorname{im} P^{c}$ and $P$ is the orthogonal projection of $L_{2}\left(\omega ; V^{\prime}\right)$ onto the subspace lin $\left\{v_{k_{0}}, \ldots, v_{k_{0}+m-1}\right\}$. Applying the projections $P$ and $P^{c}$ to (6.1) and using Theorem 3.1 we obtain that the function (6.2) is a solution of (6.1) if and only if

$$
\begin{gathered}
P F(v+w, \varepsilon)=0 \\
w-\varepsilon L_{1}^{-1} P^{c} F(v+w, \varepsilon)=0 .
\end{gathered}
$$

Thus we have split the equation (6.1) into two simpler equations (6.3) and (6.4). (They are known as the bifurcation (or determining) and the auxiliary equations, respectively.) To solve the system (6.3), (6.4) we can apply e.g. the implicit function theorem (see e.g. [11], p. 39) and obtain 
Theorem 6.3. Suppose (3.9) holds. Let the following assumptions be satisfied:

a) the equation

$$
P F\left(v_{0}, 0\right)=0
$$

has a solution $v_{0}=v_{0}^{*} \in \mathscr{U} \cap \operatorname{im} P$;

b) for some $\varrho>0$ and $\varepsilon_{0}>0 F$ maps $\left\{u ;\left\|u-v_{0}^{*}\right\|_{\mathscr{u}} \leqq \varrho\right\} \times\left[-\varepsilon_{0}, \varepsilon_{0}\right]$ continuously into $L_{2}\left(\omega ; V^{\prime}\right)$ and has a continuous partial Fréchet derivative $F_{u}^{\prime}$;

c) $\left[P F_{u}^{\prime}\left(v_{0}^{*}, 0\right)\right]^{-1} \in \mathscr{L}(\operatorname{im} P ; \mathscr{U} \cap \operatorname{im} P)$.

Then there is $\varepsilon^{*} \in\left(0, \varepsilon_{0}\right]$ such that for any $\varepsilon \in\left[-\varepsilon^{*}, \varepsilon^{*}\right]$ there is a unique $u(\varepsilon) \in \mathscr{U}$ satisfying (6.1). Moreover, $\varepsilon \rightarrow u(\varepsilon)$ is a continuous mapping of $\left[-\varepsilon^{*}, \varepsilon^{*}\right]$ into $\mathscr{U}$ and $u(0)=v_{0}^{*}$.

Remark 6.1. Since in our case $\operatorname{dim} \operatorname{ker} L_{1}<\infty$ the conditions a) and c) of the theorem can be written equivalently as follows:

$a_{1}$ ) the system of equations

$$
\int_{0}^{\omega}\left\langle F\left(\sum_{\iota=0}^{m-1} d_{0, \iota} v_{k_{0}+\iota}, 0\right), \quad v_{k_{0}+j}\right\rangle \mathrm{d} t=0, j=0, \ldots, m-1
$$

has a solution $d_{0}=d_{0}^{*}=\left(d_{0,0}^{*}, \ldots, d_{0, m-1}^{*}\right) \in \mathbb{C}^{m} ;$

$\left.\mathbf{c}_{1}\right)$ the determinant

$$
\left(\int_{0}^{\omega}\left\langle F_{u}^{\prime}\left(\sum_{\imath=0}^{m-1} d_{0, \iota}^{*} v_{k_{0}+\iota}, 0\right)\left(v_{k_{0}+j}\right), v_{k_{0}+l}\right\rangle \mathrm{d} t\right), \quad j, l=0, \ldots, m-1
$$

is non-zero.

We conclude this section with a modification of Theorem 6.1 for the operator $L_{3}$. Now, let $\mathscr{U}=L_{2}(\omega ; V) \cap W_{2}^{2}\left(\omega ; V^{\prime}\right)$.

Theorem 6.4. Suppose (3.8) holds. Let the following assumptions be satisfied:

a) $V_{1}$ and $V_{2}$ are two Hilbert spaces, $V G V_{1} G H G V_{2} G V^{\prime}$ and $V G V_{1}$ compactly, $H \mathrm{G} V_{2}$ compactly;

b) $F$ maps $L_{2}\left(\omega ; V_{1}\right) \cap W_{2}^{1}\left(\omega ; V_{2}\right)$ continuously into $L_{2}(\omega ; H)$;

c) there are two positive constants $c_{1}, c_{2}$ and $\sigma \in[0,1]$ such that

$$
\|F(u)\|_{L_{2}(\omega ; H)} \leqq c_{1}+c_{2}\|u\|_{\mathscr{U}}^{\sigma}, \quad u \in \mathscr{U}
$$

(and $c_{2}$ is sufficiently small in the case $\sigma=1$ ).

Then there is $u \in \mathscr{U}$ such that

$$
L_{3} u=F(u)
$$




\section{REGULARITY I}

Denote $\operatorname{dom} A=a^{-1} H$ and $A=a \mid \operatorname{dom} A$. In this way we have defined a linear operator

$$
A: \operatorname{dom} A \subset H \rightarrow H .
$$

Its well-known properties are listed in the following lemma.

Lemma 7.1 (see [6]).

(1) $A$ is self-adjoint,

(2) $A$ is positive definite ${ }^{*}$,

(3) $A^{-1}$ is compact.

Since $a v_{k}=\lambda_{k} v_{k}$ we have $v_{k} \in \operatorname{dom} A$ and $A v_{k}=\lambda_{k} v_{k}, k \in \mathbb{N}$.

For $s \geqq 0$ we denote by $A^{s}$ the $s$-th power of the operator $A$. Its domain of definition $\operatorname{dom} A^{s}$ is equipped with the norm

$$
\|u\|_{\operatorname{dom} A^{s}}=\left\|A^{s} u\right\|_{H}
$$

which is equivalent to the usual graph norm (for $A^{s}$ is still positive definite) and which makes dom $A^{s}$ a Hilbert space. The space dom $A^{s}$ is characterized in the following manner:

$$
\operatorname{dom} A^{s}=\left\{u ; u \in H, \sum_{k=1}^{\infty} \lambda_{k}^{2 s}\left|\left\langle u, v_{k}\right\rangle\right|^{2}<\infty\right\} .
$$

In Sections 4 and 5 we have introduced the notation $U^{0,0}, U^{1,1}$ and $U^{r, 0}$. We extend the definition to $U^{r, s}$ to any $r \geqq 0$ and $s \geqq 0$. Let

and

$$
U^{(r, s)}=W_{2}^{r}\left(\omega ; \operatorname{dom} A^{s / 2}\right)
$$

$$
U^{r, s}=U^{(r, 0)} \cap U^{(0, s)} .
$$

Lemma 7.2. Let $r, s \geqq 0$. The mapping $\mathscr{F}$ defined in (2.2) is a topological isomorphism of

(a) $U^{(r, s)}$ onto $\hat{U}^{(r, s)}$,

(b) $U^{r, s}$ onto $\hat{U}^{r, s}$.

The proof follows from Lemma 1.1 if we observe that $\left\{\omega^{-1 / 2} \lambda_{k}^{-s / 2} \mathrm{e}^{\mathrm{i} v j t} v_{k} ; j \in \mathbb{Z}\right.$, $k \in \mathbb{N}\}$ is a complete orthonormal set in $L_{2}\left(\omega ; \operatorname{dom} A^{s / 2}\right)$.

Remark 7.1. The preceding lemma and the inequality

$$
|j|^{2 \varrho} \lambda_{k}^{s(1-\varrho / r)} \leqq \text { const. }\left(|j|^{2 r}+\lambda_{k}^{s}\right)
$$

show that $U^{r, s} G U^{(\ell, s(1-\varrho / r))}$ for $\varrho \in(0, r)$. (Cf. [8], p. 19, or Lemma 8.1(5).) In particular, if $u \in U^{2,2}$ then $u^{\prime} \in L_{2}\left(\omega\right.$; $\left.\operatorname{dom} A^{1 / 2}\right)$.

*) I.e., there is $\gamma>0$ such that $\langle A u, u\rangle \geqq \gamma\|u\|_{H}^{2}, u \in \operatorname{dom} A$. 
Before stating the following propositions let us adopt the letter $P$ to denote in the critical cases the orthogonal projection of $U^{0,0}$ onto a closed subspace generated by functions from the kernel of the corresponding operator $L_{n}$, i.e., onto the subspace cl lin $\left\{\mathrm{e}^{\mathrm{i} v j t} v_{k} ;(j, k) \in \mathscr{S}_{n}\right\}$ in $U^{0,0}$ where $\mathscr{S}_{1}, \mathscr{S}_{2}$ and $\mathscr{S}_{4}$ are given by (3.11), (4.3) and (5.3), respectively, and $\mathscr{S}_{3}=\mathscr{S}_{1}$.

Theorem 7.1. 1) Let (3.8) hold. Then

2) Let (3.9) hold. Then

$$
L_{1}^{-1} \in \mathscr{L}\left(U^{0,0} ; U^{1,2}\right) .
$$

$$
L_{1}^{-1} \in \mathscr{L}\left(U^{0,0} \cap \operatorname{im} P^{c} ; U^{1,2} \cap \operatorname{im} P^{c}\right) .
$$

Proof. It is enough to prove the first assertion. If we keep the notation of Section 3 we obtain the required result from the estimate

$$
\begin{aligned}
& \|u\|_{U^{1,2}}^{2} \sim \sum_{j, k}\left(j^{2}+\lambda_{k}^{2}\right)\left|u_{j k}\right|^{2}=\sum_{j, k} \frac{j^{2}+\lambda_{k}^{2}}{v^{2} j^{2}+\left(\lambda_{k}+c\right)^{2}}\left|g_{j k}\right|^{2} \leqq \\
& \leqq \sum_{j, k} \frac{j^{2}+\lambda_{k}^{2}}{\min \left(v^{2},\left(1+c \lambda_{k}^{-1}\right)^{2}\right)\left(j^{2}+\lambda_{k}^{2}\right)}\left|g_{j k}\right|^{2} \leqq \text { const. }\|g\|_{U^{0,0}}^{2} .
\end{aligned}
$$

Theorem 7.2. 1) Let $\mathscr{S}_{2}=\emptyset$. Then

$$
L_{2}^{-1} \in \mathscr{L}\left(U^{0,0} ; U^{(1,2)} \cap U^{2,0}\right) \text {. }
$$

2) Let $\mathscr{S}_{2} \neq \emptyset$. Then

$$
L_{2}^{-1} \in \mathscr{L}\left(U^{0,0} \cap \operatorname{im} P^{c} ; U^{(1,2)} \cap U^{2,0} \cap \operatorname{im} P^{c}\right) .
$$

The proof follows from the estimate (see Lemma 4.1)

$$
\frac{\left(1+j^{2}\right) \lambda_{k}^{2}+j^{4}}{\left(-v^{2} j^{2}+\lambda_{k}+c\right)^{2}+v^{2} j^{2}\left(a+b \lambda_{k}\right)^{2}} \leqq \text { const. , } \quad(j, k) \in(\mathbb{Z} \times \mathbb{N}) \backslash \mathscr{S}_{2} .
$$

Theorem 7.3. Suppose $\alpha \in[0,1]$.

1) Let (3.8) hold. Then

2) Let (3.9) hold. Then

$$
L_{3}^{-1} \in \mathscr{L}\left(U^{(\alpha, 1-\alpha)} ; U^{2,2}\right)
$$

$$
L_{3}^{-1} \in \mathscr{L}\left(U^{(\alpha, 1-\alpha)} \cap \operatorname{im} P^{c} ; U^{2,2} \cap \operatorname{im} P^{c}\right) .
$$

Proof. By Lemma 4.1 (with $r=2$ )

$$
\frac{j^{4}+\lambda_{k}^{2}}{\left(-v^{2} j^{2}+\lambda_{k}+c\right)^{2}+a^{2} v^{2} j^{2}} \leqq \text { const. }\left(1+|j|^{2 \alpha} \lambda_{k}^{1-\alpha}\right), \quad(j, k) \in(\mathbb{Z} \times \mathbb{N}) \backslash \mathscr{S}_{3}
$$

and this estimate yields the result. 
Theorem 7.4. Suppose the condition $\left(\Lambda_{\beta}\right)$ is satisfied and $\alpha \in[-\beta, 1]$.

1) Let $\mathscr{S}_{4}=\emptyset$. Then

2) Let $\mathscr{S}_{4} \neq \emptyset$. Then

$$
L_{4}^{-1} \in \mathscr{L}\left(U^{(\beta+\alpha, 1-\alpha)} ; U^{2,2}\right) \text {. }
$$

$$
L_{4}^{-1} \in \mathscr{L}\left(U^{(\beta+\alpha, 1-\alpha)} \cap \operatorname{im} P^{c} ; U^{2,2} \cap \operatorname{im} P^{c}\right) .
$$

The proof is again an easy modification of that of Theorem 5.1. (Briefly: $\left(-v^{2} j^{2}+\right.$ $\left.+\lambda_{k}+c\right)^{-2}\left(j^{4}+\lambda_{k}^{2}\right)$ are bounded on $\Xi_{0} \cup \Xi_{1}^{\varepsilon} \cup \Xi_{2}^{\varepsilon}$ whereas on $\Xi^{\varepsilon}$ they are dominated by $|j|^{2(\beta+\alpha)} \lambda_{k}^{1-\alpha}$.)

Remark 7.2 (to Theorem 5.1). It is seen that

$$
L_{4}^{-1} \in \mathscr{L}\left(U^{(\beta-\gamma, \gamma)} ; U^{1,1} \cap W_{2}^{2}\left(\omega ; V^{\prime}\right)\right)
$$

where $\gamma \in[0, \beta]$ and similarly if $\mathscr{S}_{4} \neq \emptyset$.

Remark 7.3. The assertions of this section yield information about the "strong' solvability of the equations

$$
\begin{array}{ll}
u^{\prime}+(A+c) u=g, & \\
u^{\prime \prime}+(a+b A) u^{\prime}+(A+c) u=g, & (b \neq 0), \\
u^{\prime \prime}+a u^{\prime}+(A+c) u=g, & (a \neq 0), \\
u^{\prime \prime}+(A+c) u=g . &
\end{array}
$$

On the other hand, the propositions of Sections 3-5 can be considered as existence theorems for "weak" solutions of equations (7.3)-(7.6).

Remark 7.4. Using the above estimates one can easily establish the following ones (for $s \geqq 2$ ):

$$
\begin{gathered}
\frac{|j|^{s}+\lambda_{k}^{s}}{v^{2} j^{2}+\left(\lambda_{k}+c\right)^{2}} \leqq \text { const. }\left(|j|^{s-2}+\lambda_{k}^{s-2}\right), \quad(j, k) \in(\mathbb{Z} \times \mathbb{N}) \backslash \mathscr{S}_{1}, \\
\frac{|j|^{2 s}+\left(1+j^{2}\right) \lambda_{k}^{s}}{\left(-v^{2} j^{2}+\lambda_{k}+c\right)^{2}+v^{2} j^{2}\left(a+b \lambda_{k}\right)^{2}} \leqq \text { const. }\left(|j|^{2(s-2)}+\lambda_{k}^{s-2}\right),(j, k) \in(\mathbb{Z} \times \mathbb{N}) \backslash \mathscr{S}_{2}, \\
\frac{|j|^{2 s}+\lambda_{k}^{s}}{\left(-v^{2} j^{2}+\lambda_{k}+c\right)^{2}} \leqq \text { const. }\left(|j|^{2(s-2)}+\lambda_{k}^{s-2}\right), \quad(j, k) \in(\mathbb{Z} \times \mathbb{N}) \backslash \Xi^{\varepsilon}, \\
\frac{|j|^{2 s}+\lambda_{k}^{s}}{\left(-v^{2} j^{2}+\lambda_{k}+c\right)^{2}} \leqq \text { const. }\left(|j|^{2(\beta+\alpha)} \lambda_{k}^{s-\alpha-1}\right), \quad \alpha \in[-\beta, s-1], \quad(j, k) \in \Xi^{\varepsilon} \backslash \mathscr{S}_{4}, \\
\frac{|j|^{2 s}+\lambda_{k}^{s}}{\left(-v^{2} j^{2}+\lambda_{k}+c\right)^{2}+a^{2} v^{2} j^{2}} \leqq \text { const. }\left(|j|^{2 \alpha} \lambda_{k}^{s-\alpha-1}\right), \alpha \in[0, s-1], \quad(j, k) \in \Xi^{\varepsilon} \backslash \mathscr{S}_{3} \cdot
\end{gathered}
$$


They enable us to formulate further regularity results, e.g. if $\mathscr{S}_{n} \neq 0$ then

$$
\begin{aligned}
& L_{1}^{-1} \in \mathscr{L}\left(U^{s / 2-1, s-2} ; U^{s / 2, s}\right), \\
& L_{2}^{-1} \in \mathscr{L}\left(U^{s-2, s-2} ; U^{(1, s)} \cap U^{s, 0}\right), \\
& L_{3}^{-1} \in \mathscr{L}\left(U^{s-1, s-2} ; U^{s, s}\right), \\
& L_{4}^{-1} \in \mathscr{L}\left(U^{s+\beta-1, s-2} ; U^{s, s}\right) .
\end{aligned}
$$

More general results are obtained in the next section.

\section{REGULARITY II}

We shall investigate some further properties of the operators $L_{1}-L_{4}$. Without restricting generality we shall confine ourselves to the non-critical cases (i.e., we assume $\mathscr{S}_{n}=\emptyset$ ).

Let us recall some basic assertions concerning the Hilbert space interpolation theory which are needed in the sequel.

Lemma 8.1 (see [8]). Let $X$ and $Y$ be two separable Hilbert spaces, $X G Y$ densely. Suppose $\Theta \in[0,1]$. Then

(1) (interpolation inequality)

$$
\|u\|_{[X, Y]_{\theta}} \leqq \text { const. }\|u\|_{X}^{1-\theta}\|u\|_{Y}^{\Theta}, \quad u \in X,
$$

(2) if $0 \leqq \Theta_{0}<\Theta$, then $[X, Y]_{\Theta_{0}} G[X, Y]_{\boldsymbol{\theta}}$ densely,

(3) if $0 \leqq \Theta_{0}<\Theta_{1} \leqq 1$, then

$$
\left[[X, Y]_{\Theta_{0}},[X, Y]_{\Theta_{1}}\right]_{\theta}=[X, Y]_{(1-\theta) \theta_{0}+\theta \theta_{1}}
$$

with equivalent norms,

(4) if moreover $\mathscr{X}$ and $\mathscr{Y}$ are separable Hilbert spaces, $\mathscr{X} G \mathscr{Y}$ densely and $\pi \in \mathscr{L}(X ; \mathscr{X}) \cap \mathscr{L}(Y ; \mathscr{Y})$, then $\pi \in \mathscr{L}\left([X, Y]_{\Theta} ;[\mathscr{X}, \mathscr{Y}]_{\Theta}\right)$,

(5) if $r \geqq 0$ then

$$
L_{2}(\omega ; X) \cap W_{2}^{r}(\omega ; Y) G W_{2}^{r \theta}\left(\omega ;[X, Y]_{\theta}\right) .
$$

Suppose that $M$ is a Hilbert space,

$$
M G H \text { densely }
$$

and there is a $\Theta^{\prime} \in[0,1)$ such that $\operatorname{dom} A G[M, H]_{\theta^{\prime}}$. Put $s_{0}=2 /\left(1-\Theta^{\prime}\right)$ and define

$$
M_{s}=[M, H]_{\left(s_{0}-s\right) / s_{0}}, \quad s \in\left[0, s_{0}\right] .
$$

Hence $M_{0}=H, M_{s_{0}}=M$ and $\operatorname{dom} A A_{2}$. 
Further, suppose that

$$
A^{-1} \in \mathscr{L}\left(M_{s_{0}-2} ; M_{s_{0}}\right) .
$$

By virtue of Lemma 7.1, $A^{-1} \in \mathscr{L}\left(M_{0} ; M_{2}\right)$ and consequently by Lemma 8.1 (4)

$$
A^{-1} \in \mathscr{L}\left(M_{s-2} ; M_{s}\right), \quad s \in\left[2, s_{0}\right] .
$$

For $r, s \geqq 0, s \leqq s_{0}$ let us denote

and

$$
U_{M}^{(r, s)}=W_{2}^{r}\left(\omega ; M_{s}\right)
$$

$$
U_{M}^{r, s}=U_{M}^{(r, 0)} \cap U_{M}^{(0, s)}
$$

Theorem 8.1. Let (3.8) hold. Then

$$
L_{1}^{-1} \in \mathscr{L}\left(U_{M}^{s / 2-1, s-2} ; U_{M}^{s / 2, s}\right) \quad\left(s \in\left[2, s_{0}\right]\right) .
$$

Proof. Let us write the functions $u$ and $g$ from $U^{0,0}\left(=U_{M}^{0,0}\right)$ in the form

$$
\begin{aligned}
& u(t)=\omega^{-1 / 2} \sum_{j=-\infty}^{\infty} \mathrm{e}^{\mathrm{i} v j t} u_{j}, \quad u_{j}=\omega^{-1 / 2} \int_{0}^{\omega} \mathrm{e}^{-\mathrm{i} v j t} u(t) \mathrm{d} t, \\
& g(t)=\omega^{-1 / 2} \sum_{j=-\infty}^{\infty} \mathrm{e}^{\mathrm{i} v j t} g_{j}, \quad g_{j}=\omega^{-1 / 2} \int_{0}^{\omega} \mathrm{e}^{-\mathrm{i} v j t} g(t) \mathrm{d} t .
\end{aligned}
$$

It is a consequence of the estimate (7.1) and of the Parseval identity that

$$
\sum_{j=-\infty}^{\infty} j^{2}\left\|u_{j}\right\|_{H}^{2}+\sum_{j=-\infty}^{\infty}\left\|u_{j}\right\|_{\text {dom } A}^{2} \leqq \text { const. } \sum_{j=-\infty}^{\infty}\left\|g_{j}\right\|_{H}^{2} .
$$

In what follows, by $C$ a generic constant will be denoted, independent of $j$, but not necessarily the same in different formulas.

Thus, we have

$$
\sum_{j=-\infty}^{\infty} j^{2}\left\|u_{j}\right\|_{H}^{2} \leqq C \sum_{j=-\infty}^{\infty}\left\|g_{j}\right\|_{H}^{2}
$$

and according to $\operatorname{dom} A \mathrm{G}_{2}$

$$
\sum_{j=-\infty}^{\infty}\left\|u_{j}\right\|_{M_{2}}^{2} \leqq C \sum_{j=-\infty}^{\infty}\left\|g_{j}\right\|_{H}^{2} .
$$

The way of "regularization in $t$ " is evident. Namely, the estimate (8.3) yields for an arbitrary $s \geqq 2$

$$
\sum_{j=-\infty}^{\infty}|j|^{s}\left\|u_{j}\right\|_{H}^{2} \leqq C \sum_{j=-\infty}^{\infty}|j|^{s-2}\left\|g_{j}\right\|_{H}^{2}
$$

and by Lemma 2.2, $u \in U^{s / 2,2}$ whenever $g \in U^{s / 2-1,0}$. 
Further, for $s=2$ the result follows from (8.3) and (8.4). Let $s \in(2,4)$ (and, of course, $s \leqq s_{0}$ ). It is easy to find that $u_{j}$ satisfies the equation

$$
A u_{j}=g_{j}-\mathrm{i} v j u_{j}-c u_{j}, \quad j \in \mathbb{Z} .
$$

If $g \in U_{M}^{s / 2-1, s-2}$ then $g_{j} \in M_{s-2}, j \in \mathbb{Z}$. Since certainly $u_{j} \in M_{2}$, it is also $u_{j} \in M_{s-2}$, $j \in \mathbb{Z}$. In view of (8.2) $u_{j} \in M_{s}$ and

$$
\sum_{j=-\infty}^{\infty}\left\|u_{j}\right\|_{M_{s}}^{2} \leqq C \sum_{j=-\infty}^{\infty}\left(\left\|g_{j}\right\|_{M_{s-2}}^{2}+\left(1+j^{2}\right)\left\|u_{j}\right\|_{M_{s-2}}^{2}\right) .
$$

Using Lemma 8.1 (3), (1) and the inequality $a b \leqq p^{-1} a^{p}+q^{-1} b^{q}(a, b \geqq 0, p>1$, $\left.p^{-1}+q^{-1}=1\right)$ we obtain

$$
\begin{aligned}
\sum_{j=-\infty}^{\infty} j^{2}\left\|u_{j}\right\|_{M_{s-2}}^{2} & \leqq C \sum_{j=-\infty}^{\infty} j^{2}\left\|u_{j}\right\|_{\left[M_{2}, H\right]_{(4-s) / 2}} \leqq C \sum_{j=-\infty}^{\infty} j^{2}\left\|u_{j}\right\|_{M_{2}}^{s-2}\left\|u_{j}\right\|_{H}^{4-s} \leqq \\
& \leqq C(s) \sum_{j=-\infty}^{\infty}\left(|j|^{s-2}\left\|u_{j}\right\|_{M_{2}}^{2}+|j|^{s}\left\|u_{j}\right\|_{H}^{2}\right) .
\end{aligned}
$$

With regard to (8.3) and (8.4) we have proved for $s \in[2,4)\left(s \leqq s_{0}\right)$ the implication

$$
\begin{gathered}
g \in U^{s / 2-1, s-2} \Rightarrow \sum_{j=-\infty}^{\infty}\left\|u_{j}\right\|_{M_{s}}^{2} \leqq C \sum_{j=-\infty}^{\infty}\left(\left\|g_{j}\right\|_{M_{s-2}}^{2}+\right. \\
\left.+\left(1+|j|^{s-2}\right)\left\|g_{j}\right\|_{H}^{2}\right)<\infty .
\end{gathered}
$$

We claim that (8.8) holds for all $s$. We shall proceed by induction. Let (8.8) hold for $s \in[2,2 \sigma), \sigma \in \mathbb{N}, \sigma \geqq 2$. Let $g \in U_{M}^{s / 2-1, s-2}$ and $s \in[2 \sigma, 2(\sigma+1))\left(s \leqq s_{0}\right)$. In view of (8.2), (8.6) and by the induction hypothesis, (8.7) holds for this $s$. By the induction hypothesis

$$
\sum_{j=-\infty}^{\infty}\left\|u_{j}\right\|_{M_{s}}^{2} \leqq C \sum_{j=-\infty}^{\infty}\left(\left\|g_{j}\right\|_{M_{s-2}}^{2}+\left(1+j^{2}\right)\left\|g_{j}\right\|_{M_{s-4}}^{2}+\left(1+|j|^{s-2}\right)\left\|g_{j}\right\|_{H}^{2}\right) .
$$

In accordance with Lemma $8.1(5), g^{\prime} \in L_{2}\left(\omega ; M_{s-4}\right)$ and

$$
\sum_{j=-\infty}^{\infty}\left(1+j^{2}\right)\left\|g_{j}\right\|_{M_{s-4}}^{2} \leqq C \sum_{j=-\infty}^{\infty}\left(\left\|g_{j}\right\|_{M_{s-2}}^{2}+\left(1+|j|^{s-2}\right)\left\|g_{j}\right\|_{H}^{2}\right) .
$$

Therefore, the implication (8.8) is true for $s \in[2,2(\sigma+1))$.

Combining (8.5) and (8.8) we obtain the required result.

Remark 8.1. If moreover $A$ is a topological isomorphism of $M_{s_{0}} \cap \operatorname{dom} A$ onto $M_{s_{0}-2}$ then $L_{1}$ is a topological isomorphism of $L_{2}\left(\omega ; M_{s} \cap \operatorname{dom} A\right) \cap W_{2}^{s / 2}(\omega ; H)$ onto $U_{M}^{s / 2-1, s-2}$.

Similar theorems (and remarks) may be derived for the second-order operators $L_{2}, L_{3}$ and $L_{4}$. We state them while the proof will be given only for the operator $L_{3}$. We leave it to the reader to supply the missing details. 
Theorem 8.2. Let $\mathscr{S}_{2}=\emptyset$. Then

$$
L_{2}^{-1} \in \mathscr{L}\left(U_{M}^{s-2, s-2} ; U_{M}^{(1, s)} \cap U_{M}^{s, 0}\right) \quad\left(s \in\left[2, s_{0}\right]\right) .
$$

Theorem 8.3. Let (3.8) hold and $\alpha \in[0, s-1]$. Then

$$
L_{3}^{-1} \in \mathscr{L}\left(U_{M}^{s-1, s-2} ; U_{M}^{s, s}\right)\left(s \in\left[2, s_{0}\right]\right) \text {. }
$$

Proof. We employ the estimate

$$
\sum_{j=-\infty}^{\infty}\left(j^{4}\left\|u_{j}\right\|_{H}^{2}+\left\|u_{j}\right\|_{\text {dom } A}^{2}\right) \leqq C \sum_{j=-\infty}^{\infty}\left(1+j^{2}\right)\left\|g_{j}\right\|_{H}^{2}
$$

that is easily obtained from (7.2). Consequently,

$$
\begin{gathered}
\sum_{j=-\infty}^{\infty} j^{2}\left\|u_{j}\right\|_{H}^{2} \leqq C \sum_{j=-\infty}^{\infty}\left\|g_{j}\right\|_{H}^{2}, \\
\sum_{j=-\infty}^{\infty}\left\|u_{j}\right\|_{M_{2}}^{2} \leqq C \sum_{j=-\infty}^{\infty}\left(1+j^{2}\right)\left\|g_{j}\right\|_{H}^{2} .
\end{gathered}
$$

Evidently, $u \in U^{s, 2}$ whenever $g \in U^{s-1,0}, s \geqq 2$.

The assertion of the theorem is evident for $s=2$. Let $s \in(2,4)$. The vectors $u_{j}$ satisfy

$$
A u_{j}=g_{j}+v^{2} j^{2} u_{j}-a \mathrm{i} v j u_{j}-c u_{j}, \quad j \in \mathbb{Z} .
$$

If $g \in U_{M}^{s-1, s-2}$ then by (8.2) $u_{j} \in M_{s}$ and

$$
\sum_{j=-\infty}^{\infty}\left\|u_{j}\right\|_{M_{*}}^{2} \leqq C \sum_{j=-\infty}^{\infty}\left(\left\|g_{j}\right\|_{M_{s-2}}^{2}+\left(1+j^{4}\right)\left\|u_{j}\right\|_{M_{s-2}}^{2}\right) .
$$

Using Lemma 8.1

$$
\begin{aligned}
\sum_{j=-\infty}^{\infty} j^{4}\left\|u_{j}\right\|_{M_{s-2}}^{2} & \leqq C \sum_{j=-\infty}^{\infty} j^{4}\left\|u_{j}\right\|_{\left[M_{2}, H\right]_{(4-s) / 2}^{2} \leqq C} \sum_{j=-\infty}^{\infty} j^{4}\left\|u_{j}\right\|_{M_{2}}^{s-2}\left\|u_{j}\right\|_{H}^{4-s} \leqq \\
& \leqq C \sum_{j=-\infty}^{\infty}\left(|j|^{2(s-2)}\left\|u_{j}\right\|_{M_{2}}^{2}+|j|^{2 s}\left\|u_{j}\right\|_{H}^{2}\right) .
\end{aligned}
$$

Thus, the following implication holds for $s \in[2,4)$ :

$$
\begin{gathered}
g \in U_{M}^{s-1, s-2} \Rightarrow \sum_{j=-\infty}^{\infty}\left\|u_{j}\right\|_{M_{s}}^{2} \leqq C \sum_{j=-\infty}^{\infty}\left(\left\|g_{j}\right\|_{M_{s-2}}^{2}+\right. \\
\left.+\left(1+|j|^{2(s-1)}\right)\left\|g_{j}\right\|_{H}^{2}\right)<\infty .
\end{gathered}
$$

Let (8.13) hold for $s \in[2,2 \sigma), \sigma \in \mathbb{N}, \sigma \geqq 2$. Let $s \in[2 \sigma, 2(\sigma+1))\left(s \leqq s_{0}\right)$. (8.12) is valid owing to (8.2) and (8.11). By the hypothesis,

$$
\begin{aligned}
\sum_{j=-\infty}^{\infty}\left\|u_{j}\right\|_{M_{s}}^{2} \leqq & C \sum_{j=-\infty}^{\infty}\left(\left\|g_{j}\right\|_{M_{s-2}}^{2}+\left(1+j^{4}\right)\left\|g_{j}\right\|_{M_{s-4}}^{2}+\right. \\
& \left.+\left(1+|j|^{2(s-1)}\right)\left\|g_{j}\right\|_{H}^{2}\right) .
\end{aligned}
$$


$U_{M}^{s-2, s-2} G U_{M}^{(2, s-4)}$ by Lemma $8.1(5)$ and a fortiori, $U_{M}^{s-1, s-2} G U_{M}^{(2, s-4)}$. This implies that $(8.13)$ holds for $s \in[2,2(\sigma+1)]$. The conclusion follows readily.

Theorem 8.4. Let $\mathscr{S}_{4}=\emptyset$ and let the condition $\left(\Lambda_{\beta}\right)$ be satisfied. Then

$$
L_{4}^{-1} \in \mathscr{L}\left(U_{M}^{\beta+s-1, s-2} ; U_{M}^{s, s}\right) \quad\left(s \in\left[2, s_{0}\right]\right) .
$$

Remark 8.2. The proof of Theorem 8.3 makes use of the estimate (7.2) with $\alpha=1$. The utilization of (7.2) with $\alpha \in[0,1)$ yields another regularity result. An analogous remark holds also for Theorem 8.4.

Remark 8.3. We can choose, in particular, $M=\operatorname{dom} A^{s_{0} / 2}$ for any $s_{0} \geqq 2$. In accordance with the definition of the interpolation spaces,

$$
M_{s}=\left[\operatorname{dom} A^{s_{0} / 2}, H\right]_{\left(s_{0}-s\right) / s_{0}}=\operatorname{dom} A^{s_{0}\left(1-\left(s_{0}-s\right) / s_{0}\right) / 2}=\operatorname{dom} A^{s / 2}, \quad s \in\left[0, s_{0}\right] .
$$

Hence $U_{M}^{(r, s)}=U^{(r, s)}$ and $U_{M}^{r, s}=U^{r, s}, r, s \geqq 0$. In this particular case the results of this section coincide with those announced in Remark 7.4. When applying the theorems in this case it is required, roughly speaking, that the right hand side of the equation in question should belong (for a.e. $t$ ) to the domain of a power of the unbounded operator $A$. However, the verification need not be easy in a concrete case. Frequently we succeed in finding another suitable "regularization" space $M$ that removes the above mentioned unpleasantness (see Section 9).

Remark 8.4. The nonlinear cases can be handled also in the spaces introduced in this section by means of methods indicated in Section 6.

\section{APPLICATIONS}

The theory can be applied to the investigation of periodic solutions of partial differential equations. We show for illustration the application to the equations (7.3) - (7.6) where $A$ is an elliptic operator of the second order with boundary conditions of Dirichlet or Newton type. (The possibility of generalization to operators of order $2 m$ and other variational boundary conditions is evident.)

Let us make the following assumptions:

(9.1) $\Omega \subset \mathbb{R}^{n}$ is a bounded domain of class $C^{\infty}$,

(9.2) $a_{0}, a_{j k} \in C^{\infty}(\bar{\Omega}), a_{0}$ real-valued, $a_{j k}(x)=\overline{a_{k j}(x)}, x \in \Omega, j, k=1, \ldots, n$,

(9.3) there is $\gamma>0$ such that

$$
\sum_{j, k=1}^{n} a_{j k}(x) \xi_{j} \xi_{k} \geqq \gamma \sum_{j=1}^{n}\left|\xi_{j}\right|^{2}, \quad x \in \Omega, \quad \xi_{j} \in \mathbb{C} .
$$


Put $H=L_{2}(\Omega), \quad V=\stackrel{\circ}{W}_{2}^{1}(\Omega)$,

$$
((u, v))=\sum_{j, k=1}^{n} \int_{\Omega} a_{j k}(x) u_{x_{j}}(x) \overline{v_{x_{k}}(x)} \mathrm{d} x+\int_{\Omega} a_{0}(x) u(x) \overline{v(x)} \mathrm{d} x .
$$

In virtue of the Rellich theorem $V G H$ compactly. $((\cdot, \cdot))$ is a sesquilinear continuous symmetric form on $V \times V$ and it is $V$-elliptic provided that, for instance, $a_{0}(x) \geqq 0, x \in \Omega$.

It is easy to show that $\operatorname{dom} A$ is a subspace of $\mathscr{W}_{2}^{1}(\Omega)$ of functions $u$ for which

$$
-\sum_{j, k=1}^{n}\left(a_{j k}(x) u_{x_{j}}(x)\right)_{x_{k}}+a_{0}(x) u(x)
$$

lies in $H$. By regularity theorems for elliptic equations (see, e.g. [1], p. 129 or [9], p. 216) any such function $u$ belongs to $W_{2}^{2}(\Omega)$. Hence

$$
\operatorname{dom} A=W_{2}^{2}(\Omega) \cap \stackrel{\circ}{W}_{2}^{1}(\Omega) .
$$

Further, suppose that $\sigma$ is a real-valued function from $C^{\infty}(\partial \Omega), V=W_{2}^{1}(\Omega)$ and

$$
\begin{gathered}
((u, v))=\sum_{j, k=1}^{n} \int_{\Omega} a_{j k}(x) u_{x_{j}}(x) \overline{v_{x_{k}}(x)} \mathrm{d} x+\int_{\Omega} a_{0}(x) u(x) \overline{v(x)} \mathrm{d} x+ \\
+\int_{\partial \Omega} \sigma(x) u(x) \overline{v(x)} \mathrm{d} S .
\end{gathered}
$$

Again, $V \mathrm{G} H$ compactly and $((\cdot, \cdot))$ is a sesquilinear continuous (cf. [9], p. 15) symmetric form on $V \times V$. In addition, it is $V$-elliptic provided that

or

$$
a_{0}(x) \geqq a_{0}>0, \quad x \in \Omega, \quad \sigma(x) \geqq 0, \quad x \in \partial \Omega
$$

$$
a_{0}(x) \geqq 0, \quad \sigma(x) \geqq \sigma_{0}>0 \quad \text { on a set of positive }
$$$$
(n-1) \text {-dimensional measure }, \quad \sigma(x) \geqq 0, \quad x \in \partial \Omega \text {. }
$$

We obtain

$$
\operatorname{dom} A=\left\{u ; u \in W_{2}^{2}(\Omega), \frac{\partial u}{\partial n}+\sigma u=0 \text { on } \partial \Omega \text { (in the sense of traces) }\right\},
$$

while $A$ is given again by the formula (9.4). (Here $\partial / \partial n$ means the conormal derivative.)

In both examples the set $\operatorname{dom} A$ is closed in $W_{2}^{2}(\Omega)$ so that

$$
\left(\operatorname{dom} A,\|\cdot\|_{W_{2}^{2}(\Omega)}\right) \text { is a complete space. }
$$

Clearly, there is $c_{1}>0$ such that

$$
\|u\|_{\operatorname{dom} A}=\|A u\|_{H} \leqq c_{1}\|u\|_{W_{2}^{2}(\Omega)}, \quad u \in \operatorname{dom} A .
$$


Since $\operatorname{dom} A\left(\right.$ more precisely, $\left.\left(\operatorname{dom} A,\|\cdot\|_{\operatorname{dom} A}\right)\right)$ is a complete space, the open mapping theorem yields the existence of $c_{2}>0$ such that

$$
\|u\|_{W_{2}^{2}(\Omega)} \leqq c_{2}\|u\|_{\operatorname{dom} A}, \quad u \in \operatorname{dom} A
$$

Thus, the norms $\|\cdot\|_{\operatorname{dom} A}$ and $\|\cdot\|_{W_{2}{ }^{2}(\Omega)}$ are equivalent on $\operatorname{dom} A$. Consequently, the spaces $U^{1,2}, U^{(1,2)} \cap U^{2,0}$ and $U^{2,2}$ (which appear in Section 7) are normed equivalently by

$$
\begin{gathered}
\left(\int_{0}^{\omega} \int_{\Omega}\left(\sum_{|\alpha| \leqq 2}\left|\frac{\partial^{|\alpha|} u(t, x)}{\partial x_{1}^{\alpha_{1}} \ldots \partial x_{n}^{\alpha_{n}}}\right|^{2}+\left|\frac{\partial u(t, x)}{\partial t}\right|^{2}\right) \mathrm{d} x \mathrm{~d} t\right)^{1 / 2}, \\
\left(\int_{0}^{\omega} \int_{\Omega}\left(\sum_{|\alpha| \leqq 2, \beta \leqq 1}\left|\frac{\partial^{|\alpha|+\beta} u(t, x)}{\partial x_{1}^{\alpha_{1}} \ldots \partial x_{n}^{\alpha_{n}} \partial t^{\beta}}\right|^{2}+\left|\frac{\partial^{2} u(t, x)}{\partial t^{2}}\right|^{2}\right) \mathrm{d} x \mathrm{~d} t\right)^{1 / 2}
\end{gathered}
$$

and

$$
\left(\int_{0}^{\omega} \int_{\Omega} \sum_{|\alpha|+\beta \leqq 2}\left|\frac{\partial^{|\alpha|+\beta}}{\partial x_{1}^{\alpha_{1}} \ldots \partial x_{n}^{\alpha_{n}} \partial t^{\beta}}\right|^{2} \mathrm{~d} x \mathrm{~d} t\right)^{1 / 2},
$$

respectively.

The "regularization" space may be chosen in both cases $M=W_{2}^{s_{0}}(\Omega)$ with $s_{0} \geqq 2$ arbitrary since dom $A \mathrm{G} W_{2}^{2}(\Omega)$ (let us recall that $M_{s}=W_{2}^{s}(\Omega)$ by [8], p. 45) and $A^{-1} \in \mathscr{L}\left(W_{2}^{s_{0}-2}(\Omega) ; W_{2}^{s_{0}}(\Omega)\right)$ (see [8], p. 176). By choosing s sufficiently large we can achieve that the spaces $U_{M}^{s / 2, s}, U_{M}^{(1, s)} \cap U_{M}^{s, 0}$ and $U_{M}^{s, s}$ are embedded in the spaces of continuous functions defined on $\mathbb{R} \times \Omega$, $\omega$-periodic in $t$ and having continuous derivatives

$$
\left\{\frac{\partial u}{\partial t}, \frac{\partial^{2} u}{\partial x_{j} \partial x_{k}}\right\}, \quad\left\{\frac{\partial^{2} u}{\partial t^{2}}, \frac{\partial^{3} u}{\partial t \partial x_{j} \partial x_{k}}\right\} \text { and }\left\{\frac{\partial^{2} u}{\partial t^{2}}, \frac{\partial^{2} u}{\partial x_{j} \partial x_{k}}\right\},
$$

respectively (and obtain thereby classical solutions of $(7.3)-(7.6)$ ). This occurs by embedding theorems (see [2], p. 145) if $s>\frac{1}{2} n+3$ in the first case or $s>\frac{1}{2}(n-1)+$ +3 in the other ones.

We have often to deal with a problem given by an equation of the type (7.3)-(7.6) with $A=-\Delta$ which shows certain symmetry properties. It is then convenient to express the Laplace operator $\Delta$ e.g. in polar, cylindrical or spherical coordinates. We conclude our study by showing how to proceed in the circle-symmetrical case when the two-dimensional operator $\Delta$ is expressed in polar coordinates

$$
\left(\Delta=\frac{1}{r} \frac{\partial}{\partial r}\left(r \frac{\partial}{\partial r}\right)\right)
$$

We shall work in weight spaces and for this purpose let us introduce the following definition. 
By the symbol $L_{2, r}(0,1)$ we denote the space of (classes of) complex-valued functions defined on $(0,1)$ for which

$$
\|u\|_{L_{2, r}(0,1)}=\left(\int_{0}^{1} r|u(r)|^{2} \mathrm{~d} r\right)^{1 / 2}<\infty .
$$

$L_{2, r}(0,1)$ is a Hilbert space with the inner product

$$
\langle u, v\rangle=\int_{0}^{1} r u(r) \overline{v(r)} \mathrm{d} r .
$$

Put $H=L_{2, r}(0,1)$ and $V=\left\{u ; u, u^{\prime} \in L_{2, r}(0,1), u(1)=0\right\} . V$ is a Hilbert space with the inner product

$$
\langle u, v\rangle_{V}=\int_{0}^{1} r\left(u(r) \overline{v(r)}+u^{\prime}(r) \overline{v^{\prime}(r)}\right) \mathrm{d} r .
$$

Obviously, $V \mathrm{G} H$ densely.

Further, let

$$
((u, v))=\int_{0}^{1} r u^{\prime}(r) \overline{v^{\prime}(r)} \mathrm{d} r, \quad u, v \in V .
$$

Evidently, $((\cdot, \cdot))$ is a sesquilinear continuous form on $V \times V$. In order to prove the $V$-ellipticity we write for $u \in V$

$$
u(r)=-\int_{r}^{1} u^{\prime}(s) \mathrm{d} s, \quad r \in(0,1),
$$

hence

$$
|u(r)|^{2} \leqq \int_{r}^{1} s\left|u^{\prime}(s)\right|^{2} \mathrm{~d} s \int_{r}^{1} s^{-1} \mathrm{~d} s=|\ln r| \int_{r}^{1} s\left|u^{\prime}(s)\right|^{2} \mathrm{~d} s .
$$

Thus,

$$
\int_{0}^{1} r|u(r)|^{2} \mathrm{~d} r \leqq \int_{0}^{1}|u(r)|^{2} \mathrm{~d} r \leqq \text { const. } \int_{0}^{1} r\left|u^{\prime}(r)\right|^{2} \mathrm{~d} r
$$

and we can check easily that there is $c>0$ such that $((u, u)) \geqq c\|u\|_{V}^{2}, u \in V$.

It remains to show that $V \mathrm{G} H$ compactly (cf. [14], p. 354). It is immediate that $V G W_{2}^{1}(\delta, 1)$ for any $\delta \in(0,1)$ and by the Rellich theorem $W_{2}^{1}(\delta, 1) G L_{2}(\delta, 1)$ compactly. Since $L_{2, r}(\delta, 1)=L_{2}(\delta, 1)$ (with equivalent norms) the embedding $V \mathrm{G} L_{2, r}(\delta, 1)$ is also compact. Let $u_{m}$ be a bounded sequence in $V$. In view of the reflexivity of $V$ we can assume that $u_{m} \rightarrow u$ weakly in $V$ (replacing $u_{m}$ by a subsequence if necessary). We prove that $u_{m} \rightarrow u$ in $L_{2, r}(0,1)$ (strongly). We let $\chi_{\delta}$ be a function defined by $\chi_{\delta}(r)=0,0 \leqq r<\delta, \chi_{\delta}(r)=1, \delta \leqq r<1$. Then $\chi_{\delta} u_{m}$ is convergent in $L_{2, r}(0,1)$. Because of the inequality

$$
\begin{gathered}
\quad\left\|u_{m}-u_{n}\right\|_{L_{2, r}(0,1)} \leqq\left\|u_{m}-\chi_{\delta} u_{m}\right\|_{L_{2, r}(0,1)}+ \\
+\left\|\chi_{\delta} u_{m}-\chi_{\delta} u_{n}\right\|_{L_{2, r}(0,1)}+\left\|\chi_{\delta} u_{n}-u_{n}\right\|_{L_{2, r}(0,1)}
\end{gathered}
$$


if suffices to show that $\left\|u_{m}-\chi_{\delta} u_{m}\right\|_{L_{2, r}(0,1)} \rightarrow 0$ as $\delta \rightarrow 0+$ uniformly with respect to $m$. But this is a consequence of the estimates

$$
\begin{gathered}
\left\|u_{m}-\chi_{\delta} u_{m}\right\|_{L_{2, r}(0,1)}^{2}=\int_{0}^{\delta} r\left|u_{m}(r)\right|^{2} \mathrm{~d} r \leqq \delta \int_{0}^{\delta}\left|u_{m}(r)\right|^{2} \mathrm{~d} r \leqq \\
\leqq \text { const. } \delta \int_{0}^{1} r\left|u_{m}^{\prime}(r)\right|^{2} \mathrm{~d} r \leqq \text { const. } \delta\left\|u_{m}\right\|_{V}^{2} .
\end{gathered}
$$

We have thereby verified that $V \mathrm{G} H$ compactly.

In this example $\lambda_{k}=\mu_{k}^{2}, v_{k}=N_{k} J_{0}\left(\mu_{k} r\right)$, where $J_{0}$ is the Bessel function of order zero, $\mu_{k}$ are positive roots of $J_{0}$ (each eigenvalue is simple) and $N_{k}$ are the normalization constants.

\section{References}

[1] S. Agmon: Lectures on elliptic boundary value problems. Van Nostrand, Princeton-New Jersey 1965 .

[2] O. V. Bèsov, V. P. Iljin, S. M. Nikol'skij: Integral representations of functions and embedding theorems. (Russian.) Izd. Nauka, Moskva 1975.

[3] A. I. Hinčin: Continued fractions. (Russian.) Fizmatgiz, Moskva 1961.

[4] A. Kufner, J. Kadlec: Fourier series. Academia, Praha 1971.

[5] O. A. Ladyženskaja: Mixed problem for a hyperbolic equation. (Russian.) Gos. izd. teh.-teor. lit., Moskva 1953.

[6] J.-L. Lions: Equations différentielles opérationnelles et problèmes aux limites. SpringerVerlag, Berlin-Göttingen-Heidelberg 1961.

[7] J.-L. Lions: Quelques méthodes de résolution des problèmes aux limites non linéaires. Dunod, Paris 1969.

[8] J.-L. Lions, E. Magenes: Problèmes aux limites non homogenes et applications I. Dunod, Paris 1968.

[9] J. Nečas: Les méthodes directes en théorie des équations elliptiques. Academia, Praha 1967.

[10] B. Novák: Remark on periodic solutions of linear wave equation in one dimension. Comment. Math. Univ. Carolinae 15 (1974), 513-519.

[11] G. Prodi, A. Ambrosetti: Analisi nonlineare. Scuola normale superiore, Pisa 1973.

[12] $P$. H. Rabinowitz: Periodic solutions of nonlinear hyperbolic partial differential equations. Comm. Pure Appl. Math. 20 (1967), 145-205.

[13] I. Straškraba: Existence and uniqueness of periodic solutions of linear differential equations in Banach spaces. Czechoslovak Math. J. (to appear).

[14] H. Triebel: Höhere Analysis. VEB, Berlin 1972.

[15] O. Vejvoda et al.: Partial differential equations: time periodic solutions. Sijthoff-Nordhoff (to appear).

Author's address: 11567 Praha 1, Žitná 25, ČSSR (Matematický ústav ČSAV). 